\title{
A Systematic Review of Worldwide Consumption of Ultra-Processed Foods: Findings and Criticisms
}

\author{
Mirko Marino (D), Federica Puppo, Cristian Del Bo’*(D), Valentina Vinelli, Patrizia Riso ${ }^{(D)}$, Marisa Porrini ${ }^{+}(\mathbb{D})$ and \\ Daniela Martini ${ }^{+}(\mathbb{D}$
}

check for updates

Citation: Marino, M.; Puppo, F.; Del Bo', C.; Vinelli, V.; Riso, P.; Porrini, M.; Martini, D. A Systematic Review of Worldwide Consumption of Ultra-Processed Foods: Findings and Criticisms. Nutrients 2021, 13, 2778. https://doi.org/10.3390/nu13082778

Academic Editor: Alessandro Leone

Received: 30 June 2021

Accepted: 11 August 2021

Published: 13 August 2021

Publisher's Note: MDPI stays neutral with regard to jurisdictional claims in published maps and institutional affiliations.

Copyright: (c) 2021 by the authors. Licensee MDPI, Basel, Switzerland. This article is an open access article distributed under the terms and conditions of the Creative Commons Attribution (CC BY) license (https:/ / creativecommons.org/licenses/by/ $4.0 /)$.
Department of Food, Environmental and Nutritional Sciences (DeFENS), Università degli Studi di Milano, 20123 Milan, Italy; mirko.marino@unimi.it (M.M.); federica.puppo@studenti.unimi.it (F.P.); valentina.vinelli@unimi.it (V.V.); patrizia.riso@unimi.it (P.R.); marisa.porrini@unimi.it (M.P.); daniela.martini@unimi.it (D.M.)

* Correspondence: cristian.delbo@unimi.it

+ These authors contributed equally to this work.

Abstract: A debate is ongoing on the significance and appropriateness of the NOVA classification as a tool for categorizing foods based on their degree of processing. As such, the role of ultraprocessed food (UPF) on human health is still not completely understood. With this review, we aimed to investigate the actual level of consumption of UPF across countries and target populations to determine the impact in real contexts. Suitable articles published up to March 2021 were sourced through the PubMed and SCOPUS databases. Overall, 99 studies providing data on the level of UPF consumption expressed as the percentage of total energy intake were identified, for a total of $1,378,454$ participants. Most of them were published in Brazil $(n=38)$ and the United States $(n=15)$, and the $24 \mathrm{~h}$ recall was the most-used tool $(n=63)$. Analysis of the results revealed that the United States and the United Kingdom were the countries with the highest percent energy intake from UPF (generally $>50 \%$ ), whereas Italy had the lowest levels (about $10 \%$ ); the latter was inversely associated with adherence to the Mediterranean diet. High variability was also observed based on sex, age, and body mass index, with men, young people, and overweight/obese subjects generally having higher levels of consumption compared to older subjects. Overall, our findings underline the large differences in UPF intake. Since most of the observations derived from studies conducted with food questionnaires are not specifically validated for UPF, further efforts are essential to confirm the results previously obtained and to investigate further the association between UPF consumption and health status, also considering the actual contribution within different dietary patterns, which has been less investigated to date.

Keywords: dietary habits; dietary intake; human health; NOVA group; processing; food technology; food consumption

\section{Introduction}

Food processing includes all the processes needed to transform raw or harvested foodstuffs into new products, ensuring their safety, palatability, and shelf-life [1]. However, in recent decades, food processing has drastically changed to address consumer preferences. The demand for food items with longer shelf-life and improved palatability has led to other natural or artificial ingredients being added to processed foods, which may, to some extent, impact the nutritional quality of these foods, which are often characterized by high sugar, fat, and salt contents [2]. For this reason, interest is growing in elucidating whether the high consumption of these foods negatively impacts diet quality and, in turn, human health.

One of the main limits to the evaluation of the role and impact of food processing on health status is the lack of a proper definition and classification of "processed food". In 2009, the European Prospective Investigation into Cancer and Nutrition (EPIC) [3] proposed a 
classification of foods into three main categories: highly processed, moderately processed, and non-processed foods. A Brazilian group of researchers coordinated by Monteiro instead proposed the NOVA classification, which classifies foods into four main categories based on their degree of processing, without providing any indication of their nutritional content: (i) unprocessed and minimally processed foods such as fruit and vegetables, milk, eggs, and meats; (ii) processed culinary ingredients, including oils, butter, lard, sugar, and salt; (iii) processed foods such as canned fish or legumes, typically produced by adding salt, oil, sugar, or other substances from group 2 to group 1 foods, and using preservation methods such as canning and bottling; and (iv) ultra-processed foods (UPF) [4]. The latter comprises formulations of ingredients, mostly for exclusive industrial use, produced through a series of industrial processes that, for instance, include the fractioning of whole foods into substances, the assembly of unmodified and modified food substances, and the frequent use of cosmetic additives, often added to improve the sensory characteristics of the final product [5]. UPF include, amongst others, carbonated soft drinks; sweet, fatty, or salty packaged snacks; candies; ice creams; pastries; margarines; and many others.

The Siga classification further attempts to categorize foods based on processing, which was developed by combining the four holistic NOVA groups with four additional new reductionist subgroups that consider the impact of processing on the food/ingredient matrix; the contents of added salt, sugar, and fat; the nature and number of markers of ultra-processing; and the levels of at-risk additives [6].

Despite the different types of food classification systems based on the degree of processing, the NOVA classification is the most widely used worldwide by researchers. Since it was proposed, the NOVA classification has been used in many epidemiological studies to investigate the association between the levels of UPF consumption and diet quality $[7,8]$ and/or the potential effect of UPF consumption on human health. For instance, a narrative review summarized the main findings from observational studies reporting a higher risk of all-cause mortality with high consumption of UPF [9]. In another narrative review, high UPF consumption was associated with an increased risk of all-cause mortality including cardiovascular diseases, coronary heart diseases, cerebrovascular diseases, cancer risk, and numerous other metabolic diseases [10]. More recently, a systematic review and meta-analysis of observational studies highlighted that increased UPF consumption is associated with a worse cardiometabolic risk profile and a higher risk of cardiovascular diseases and all-cause mortality [11]. Furthermore, the results of a randomized controlled trial showed that a two-week intervention with a diet rich in UPF consumed ad libitum resulted in significantly increased weight gain amongst American adults compared to a diet of unprocessed foods [12].

The criteria used in these types of classification are often ambiguous, only based on a chaotic conception of processing that is not only related to technical processes [13]. Notably, the dietary habits of different populations may vary widely based on tradition, culture, and individual characteristics; in turn, the consumption of UPF in terms of type and amount may differ across target populations and countries.

Based on these premises, understanding the implications of increasing UPF consumption for global human health is of upmost importance, together with the identification of the cause-effect relationship. In order to pursue this important goal, it is first of all crucial to estimate/quantify the amount of UPF consumed adequately. The aim of the present review was to investigate the levels of UPF consumption systematically-as defined by the NOVA system-across countries around the world. The summarized information will be useful to provide a clear overview of the actual consumption of UPF in different countries, of their contribution to total energy intake also in diverse target groups (i.e., children, adolescents, adults), but also to bring out possible criticisms in the estimation of UPF intake and to underline eventual gaps that need to be considered in future studies. 


\section{Materials and Methods}

\subsection{Search Strategy and Study Selection}

This study was conducted according to the Preferred Reporting Items for Systematic Reviews Meta-Analysis (PRISMA) guidelines. To identify pertinent articles, we searched the PubMed and SCOPUS databases up to March 2021 using the combination of the following keywords: ("ultraprocessed food" OR "ultra-processed food" OR "NOVA system" OR "NOVA classification" OR "minimally processed food") AND ("intake" OR "consumption"). Reference lists of included manuscripts and relevant reviews were examined for any possible unidentified study.

Studies were included if they: (i) provided information on UPF consumption expressed as the percentage of energy for each NOVA category with respect to total dietary intake; (ii) provided information for the general population or for at least one target group; (iii) were published in the English language. Conversely, studies were excluded if they: (i) were published before 2009; (ii) did not use the NOVA classification; (iii) were systematic reviews or meta-analyses; (iv) expressed UPF intake as tertiles/quartiles without providing information about the net consumption.

\subsection{Data Extraction and Presentation}

Data from included papers were extracted by two reviewers (M.M. and F.P.). Any discrepancy between the reviewers was resolved through consultation with a third independent author (C.D.B) to achieve consensus. The following information is reported: (i) authors and year of publication; (ii) country; (iii) characteristics of the population under study; (iv) method of dietary assessment; (v) results expressed as a percentage of energy from UPF compared to total energy intake. Studies are summarized in different tables showing the percentage of energy intake (TEI) from UPF compared to total energy intake, considering the main factors such as age, sex, and BMI. Data are reported as the mean or median value followed by the standard deviation (SD), standard error of the mean (SEM), confidence interval (CI), or interquartile range (IQR). When available, the statistical significance is reported.

\section{Results}

\subsection{Study Selection}

A total of 1051 studies, conducted between 2009 and 2021, were identified using the PubMed and SCOPUS databases. After eliminating 414 duplicates, 434 articles were excluded based on the titles and abstracts, and 97 studies were removed based on the full-text assessment. A total of 106 articles met the inclusion criteria so were included in the final analysis. Among these, five studies led to two different articles each, which were therefore considered as unique studies. Thus, at the end of the process, a total of 99 unique studies were included (Figure 1). 


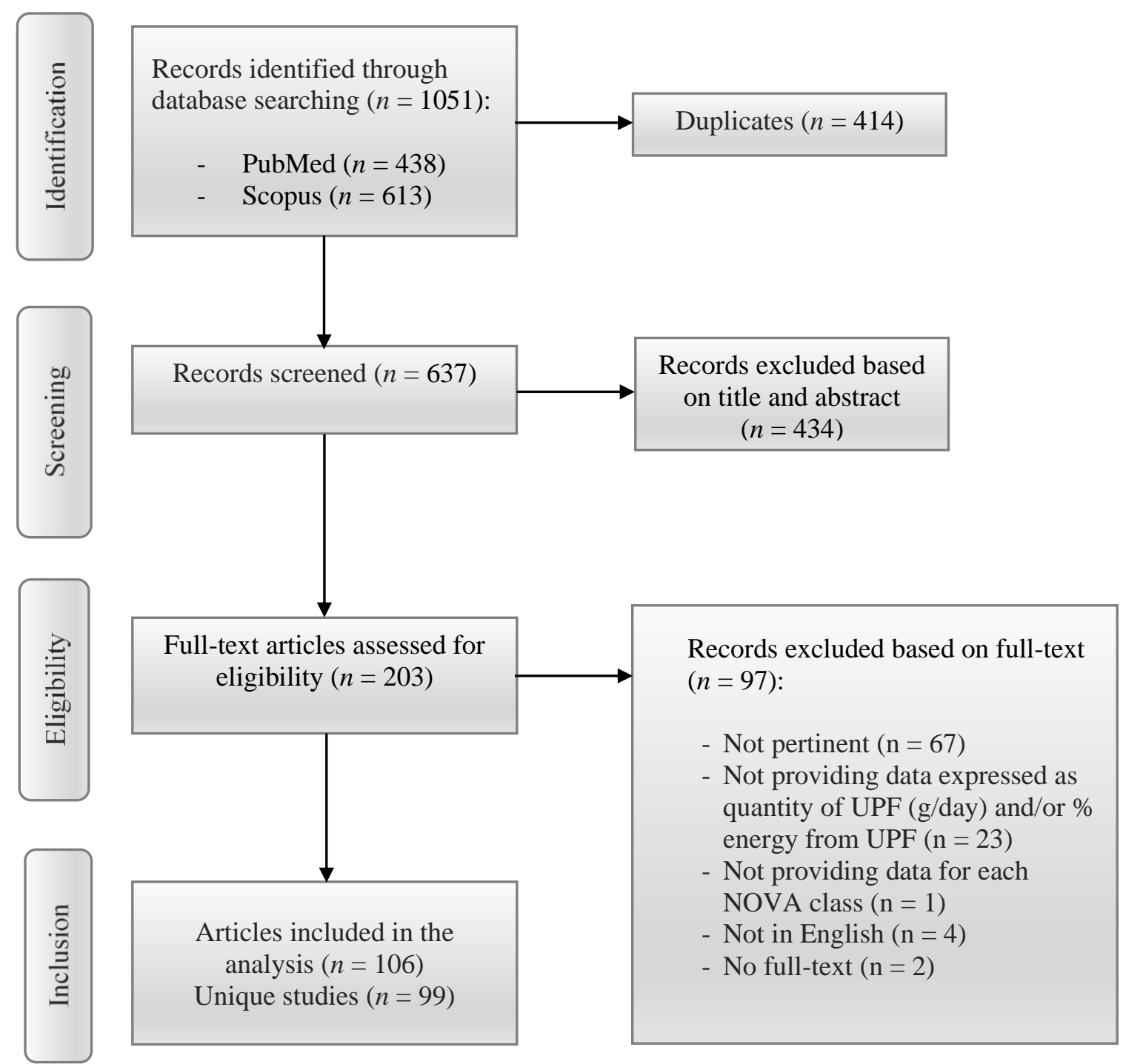

Figure 1. PRISMA flow diagram of the included studies.

\subsection{Characteristics of the Studies}

Among the 99 studies, a total of 1,378,454 subjects were considered, with the number of subjects ranging from 40 [14] to 110,260 [15]. On average, the age of participants was 38.9 years, although half of the studies did not report this information. Fifteen studies focused only on children [16-30], seven on adolescents [31-37], five on pregnant women [38-42], and three on older subjects [43-45], whereas others focused on an adult population or on $\geq 2$ target groups. Overall, $58.8 \%$ of participants were women.

The number of studies performed in different countries is reported in Figure 2. As shown, Brazil had the highest number of studies $(n=38)$, followed by the United States $(n=15)$, France $(n=8)$, the United Kingdom $(n=6)$, Canada $(n=6)$, and Spain $(n=4)$. Fewer studies were performed in Malaysia, $(n=3)$, and in other countries such as Mexico, Italy, Australia, Korea, and Portugal $(n=2)$; only one study each was conducted in Chile, Japan, Indonesia, Lebanon, Israel, the Netherlands, Colombia, Belgium, and New Zealand. 


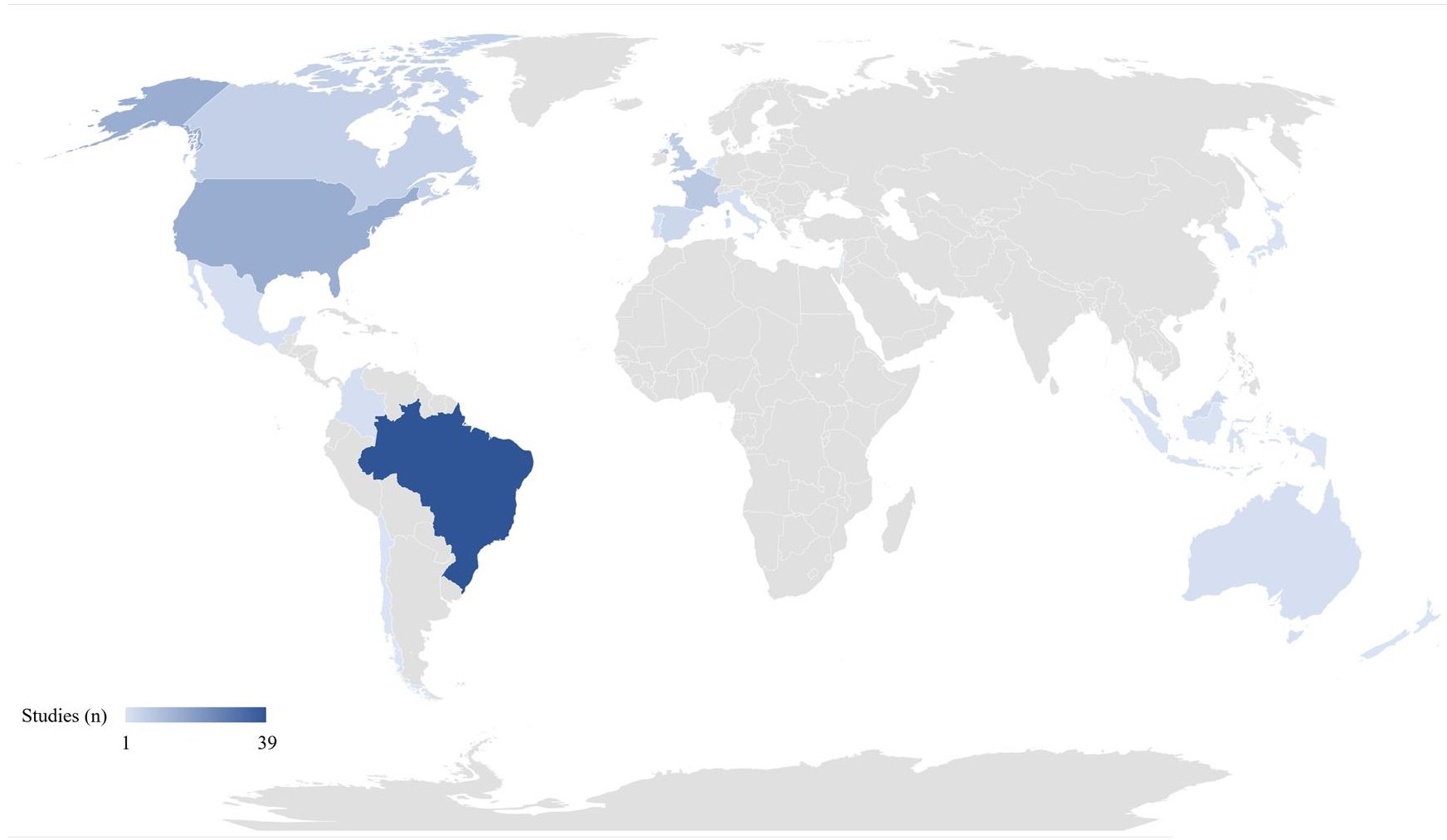

Figure 2. Countries in which studies on consumption levels for UPF were performed; the darker the blue, the higher the number of studies conducted.

Regarding the method of dietary assessment, the $24 \mathrm{~h}$ recall was the most-used tool $(n=62)$, followed by food-frequency questionnaires (FFQs) $(n=28)$ and food diaries $(n=10)$.

\subsection{Levels of UPF Intake}

The levels of consumption of UPF shown in the retrieved studies are reported in Table 1, while results stratified for specific target groups are provided in Tables 2-4.

The data revealed a high variability in terms of the percentage (\%) of energy provided by the consumption of food belonging to group 4 based on the NOVA classification. Similar findings were observed both intra- and inter-country. The highest levels of consumption were observed in the United States and the United Kingdom with the percent of energy intake generally higher than $50 \%$ with respect to TEI $[18,19,36,38,46-54]$. Conversely, the lowest levels were observed in Italy, in which the two studies identified reported about $10 \%$ energy obtained from UPF $[55,56]$.

Regarding the variability within the same country, Brazil showed levels of UPF consumption ranging from $7.7 \%$ in 64 subjects aged $25-57$ years [57] to $51.2 \%$ of total energy intake in over 4200 subjects [58]. A lower variability was identified in studies performed in the United States, with levels of intake ranging from $50 \%$ to $70 \%$, with the only exception being a study observing an energy intake of $35.5 \%$ in almost 92,000 subjects aged 55-74 years [59]. 


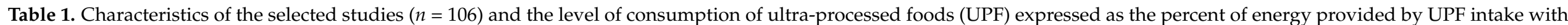
respect to total energy intake (TEI).

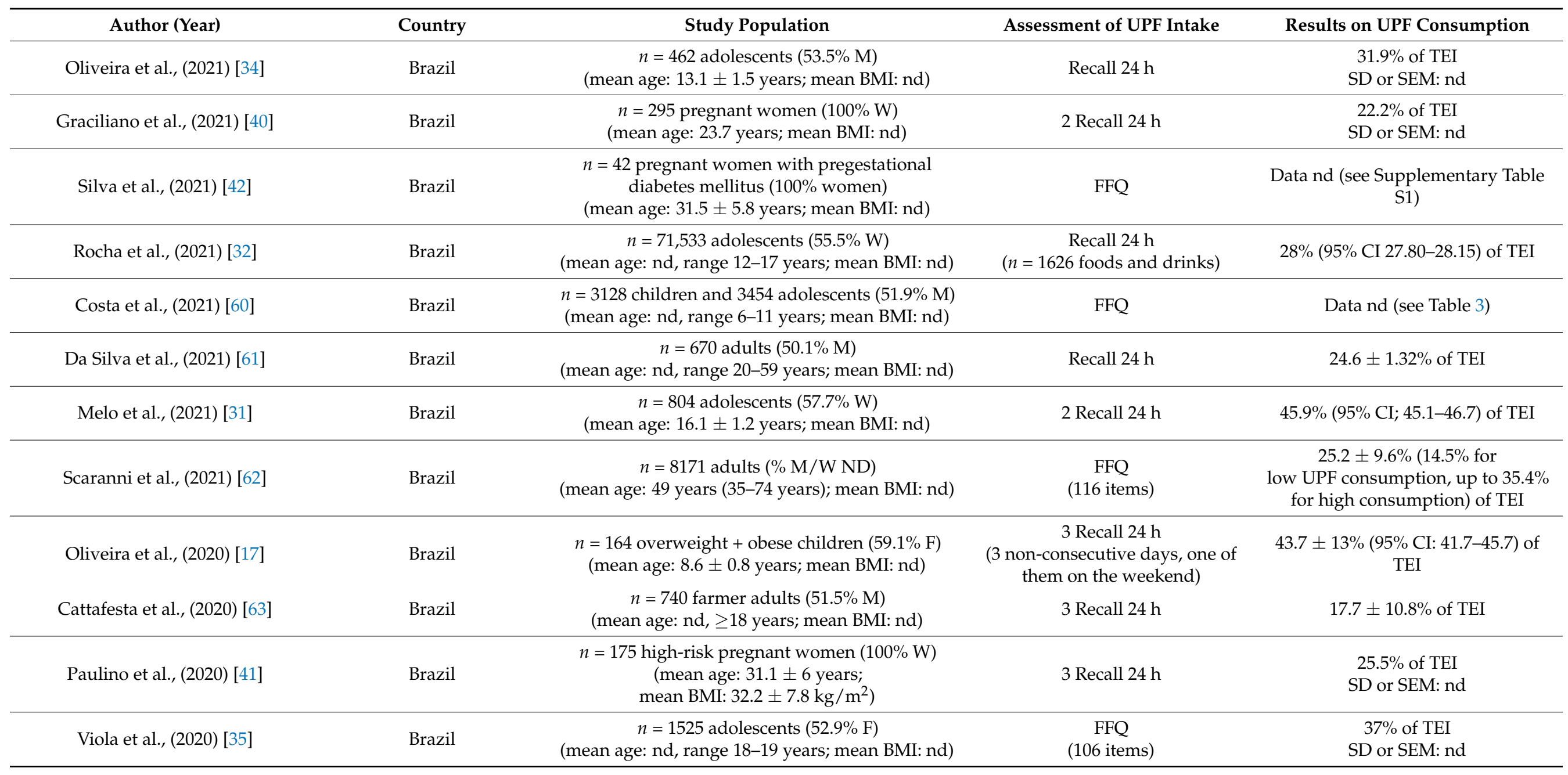


Table 1. Cont.

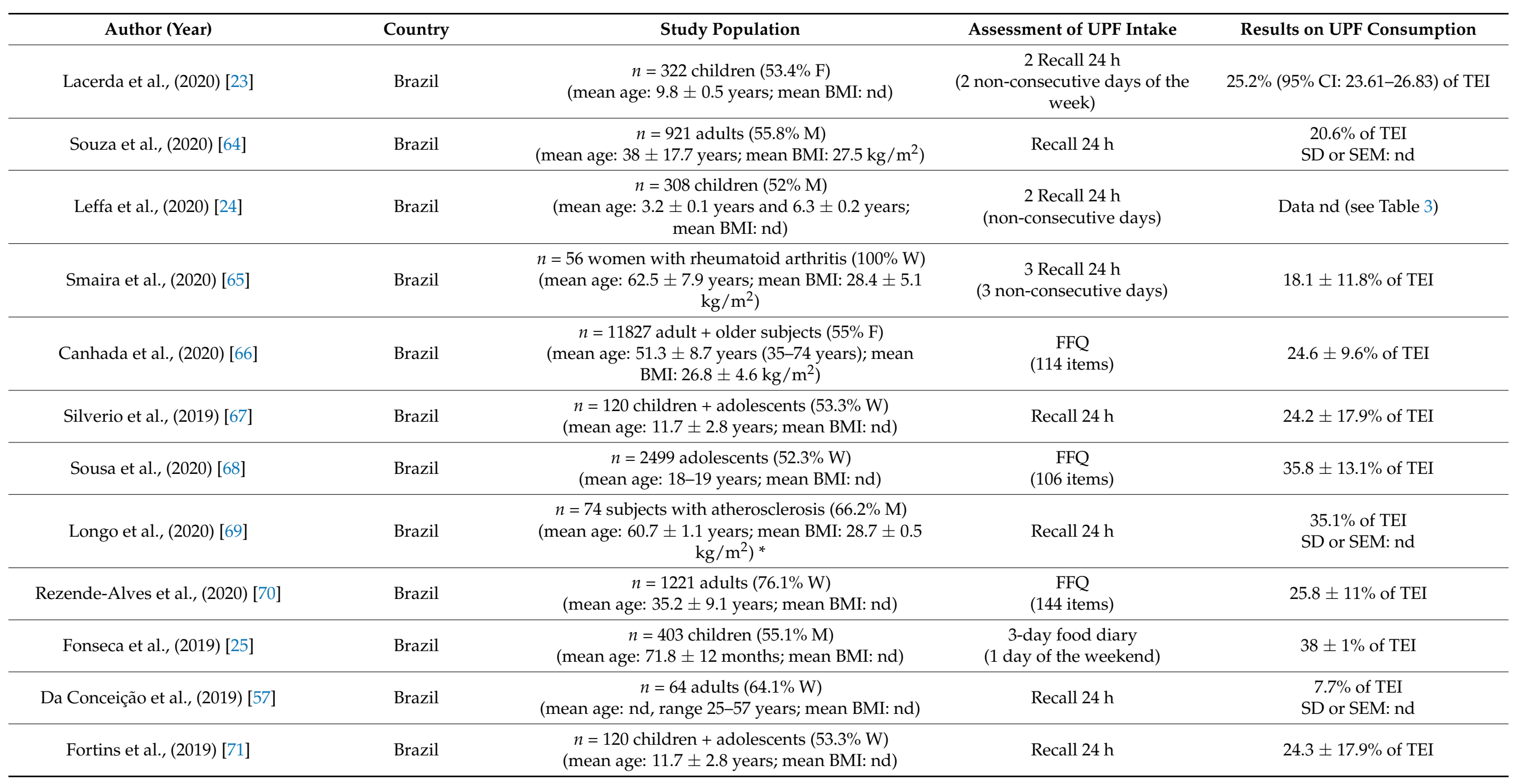


Table 1. Cont

\begin{tabular}{|c|c|c|c|c|}
\hline Author (Year) & Country & Study Population & Assessment of UPF Intake & Results on UPF Consumption \\
\hline Ferreira et al., (2019) [26] & Brazil & $\begin{aligned} n= & 206 \text { children }+ \text { adolescents }(53 \% \mathrm{~W}) \\
& (\text { age }>10 \text { years; mean BMI: } \mathrm{nd})\end{aligned}$ & Recall $24 \mathrm{~h}$ & $\begin{array}{l}31 \% \text { of TEI } \\
\text { SD or SEM: nd }\end{array}$ \\
\hline Gomes et al., (2019) [39] & Brazil & $\begin{array}{l}n=353 \text { pregnant women }(100 \% \mathrm{~W}) \\
\text { (mean age: } \mathrm{nd} \text {; mean BMI: } \mathrm{nd})\end{array}$ & 2 Recall $24 \mathrm{~h}$ & $\begin{array}{l}24.6 \% \text { of TEI } \\
\text { SD or SEM: nd }\end{array}$ \\
\hline Enes et al., (2019) [37] & Brazil & $\begin{array}{l}n=200 \text { adolescents }(56 \% \text { F) } \\
\text { (mean age: nd, range } 10-18 \text { years; mean BMI: nd) }\end{array}$ & FFQ & $50.6 \pm 1.0 \% *$ of TEI \\
\hline Silva et al., (2018) [72] & Brazil & $\begin{array}{l}n=8977 \text { adult }+ \text { older subjects }(51.9 \% \mathrm{~W} \text { ) } \\
\text { (mean age: nd, range 35-64 years; mean BMI: nd) }\end{array}$ & $\begin{array}{c}\text { FFQ } \\
\text { (114 items) } \\
\end{array}$ & $\begin{array}{l}22.7 \% \text { of TEI } \\
\text { SD or SEM: nd }\end{array}$ \\
\hline Simões et al., (2018) [73] & Brazil & $\begin{array}{c}n=14378 \text { adult }+ \text { older subjects }(54.2 \% \text { W) } \\
\text { (mean age: nd, range } 35-74 \text { years; mean BMI: nd) }\end{array}$ & $\begin{array}{c}\text { FFQ } \\
\text { (114 items) }\end{array}$ & $\begin{array}{l}22.7 \% \text { of TEI } \\
\text { SD or SEM: nd }\end{array}$ \\
\hline Louzada et al., (2018) [74] & Brazil & $\begin{array}{c}n=32,898 \text { subjects (\% M/W: } \mathrm{nd} \text { ) } \\
\text { (mean age: } \mathrm{nd}, \geq 10 \text { years; mean BMI: nd) }\end{array}$ & 2 Recall $24 \mathrm{~h}$ & $\begin{array}{l}20.4 \% \text { of TEI } \\
\text { SD or SEM: nd }\end{array}$ \\
\hline Bielemann et al., (2018) [28] & Brazil & $\begin{array}{c}n=3427 \text { children }(51.9 \% \mathrm{M}) \\
\text { (mean age: } 6 \text { years; mean BMI: nd) }\end{array}$ & $\begin{array}{c}\text { FFQ } \\
\text { (54 items) }\end{array}$ & $40.3 \pm 11.7 \%$ of TEI \\
\hline D'Avila et al., (2017) [33] & Brazil & $\begin{array}{c}n=784 \text { adolescents }(57.4 \% \mathrm{~W}) \\
\text { (mean age: } 15.2 \pm 1.3 \text { years; mean BMI: nd) }\end{array}$ & $\begin{array}{c}\text { FFQ } \\
\text { (90 items) }\end{array}$ & $\begin{array}{l}49.2 \% \text { of TEI } \\
\text { SD or SEM: nd }\end{array}$ \\
\hline Batalha et al., (2017) [27] & Brazil & $\begin{array}{l}\quad n=1185 \text { children }(51.2 \% \mathrm{M}) \\
\text { (mean age: nd, range } 15-35 \text { mo.; mean BMI: nd) }\end{array}$ & Recall $24 \mathrm{~h}$ & $\begin{array}{l}24.5 \% \text { of TEI } \\
\text { SD or SEM: nd }\end{array}$ \\
\hline Karnopp et al., (2017) [29] & Brazil & $\begin{array}{l}\quad n=770 \text { children }(52 \% \mathrm{M}) \\
\text { (mean age: nd, range } 0-72 \text { mo; mean BMI: nd) }\end{array}$ & Recall $24 \mathrm{~h}$ & $\begin{array}{l}32 \% \text { of TEI } \\
\text { SD or SEM: nd }\end{array}$ \\
\hline Bielemann et al., (2015) [58] & Brazil & $\begin{array}{c}n=4202 \text { adults }(51.4 \% \mathrm{M}) \\
\text { (mean age: } 21.8 \text { years; mean BMI: nd) }\end{array}$ & FFQ & $51.2 \%$ (95\% CI: 50.8-51.6) of TEI \\
\hline Sparrenberger et al., (2015) [30] & Brazil & $\begin{array}{l}n=204 \text { children ( } 50 \% \text { girls) } \\
\text { (mean age: } 5.9 \pm 2.5 \text { years; mean BMI: nd) }\end{array}$ & 2 Recall $24 \mathrm{~h}$ & $47 \pm 1.1 \% *$ of TEI \\
\hline Louzada et al., (2015a) [75] & Brazil & $\begin{array}{c}n=32,898 \text { subjects (\% M/F: } \mathrm{nd} \text { ) } \\
\text { (mean age: } \mathrm{nd}, \geq 10 \text { years; mean BMI: } \mathrm{nd} \text { ) }\end{array}$ & 2 Recall $24 \mathrm{~h}$ & $\begin{array}{l}21.5 \% \text { of TEI } \\
\text { SD or SEM: nd }\end{array}$ \\
\hline Louzada et al., (2015b) [76] & Brazil & $\begin{array}{c}n=30,243 \text { subjects }(50.2 \% \mathrm{~W}) \\
\text { (mean age: } \mathrm{nd}, \geq 10 \text { years; mean BMI: nd) }\end{array}$ & 2 Recall $24 \mathrm{~h}$ & $\begin{array}{l}29.6 \% \text { of TEI } \\
\text { SD or SEM: nd }\end{array}$ \\
\hline
\end{tabular}


Table 1. Cont.

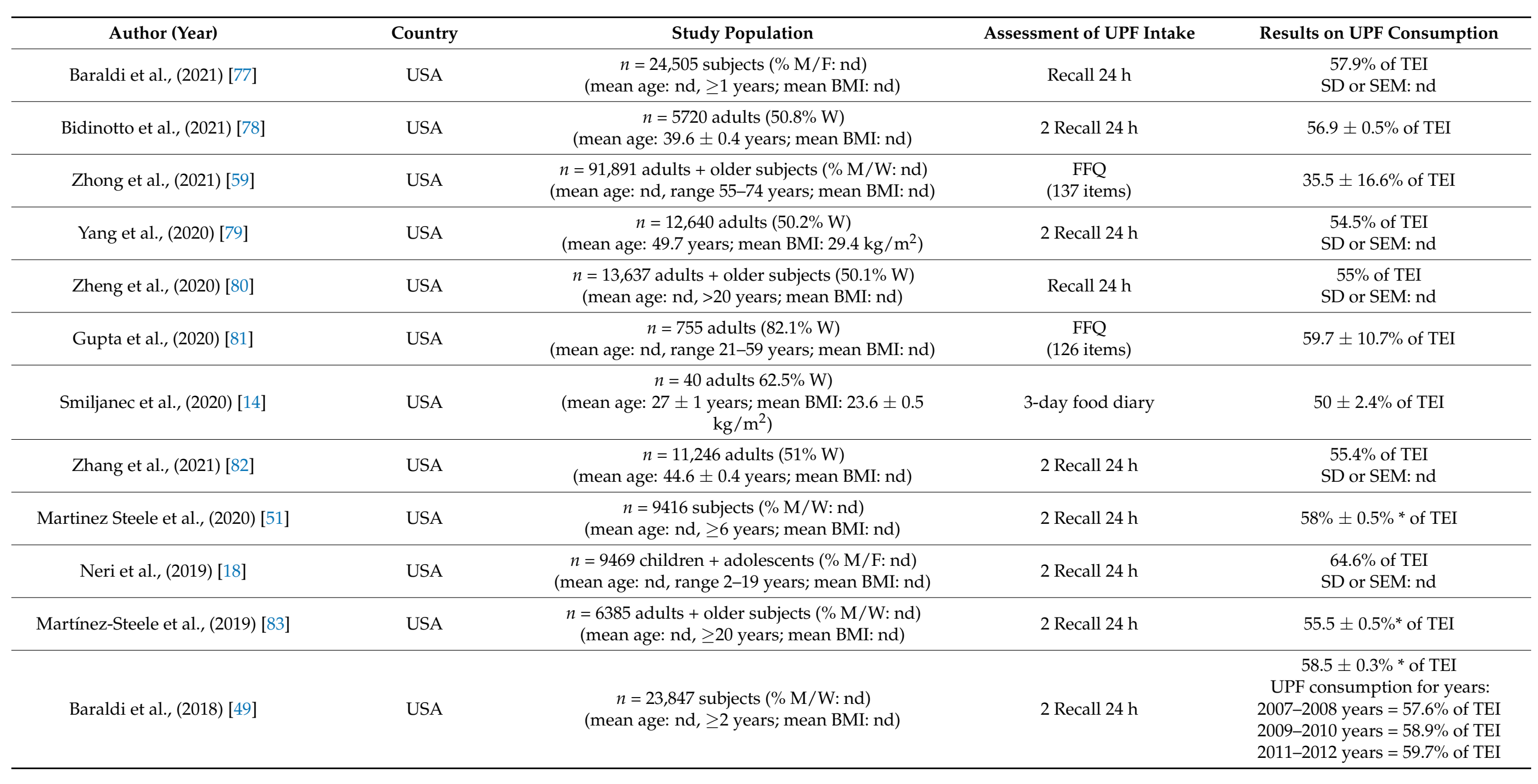


Table 1. Cont.

\begin{tabular}{|c|c|c|c|c|}
\hline Author (Year) & Country & Study Population & Assessment of UPF Intake & Results on UPF Consumption \\
\hline Juul et al., (2018) [52] & USA & $\begin{array}{c}n=15,977 \text { adults }(50.6 \% \mathrm{~W}) \\
\text { (mean age: } 41.9 \pm 0.2 \text { years; mean BMI: } 28.9 \pm 0.1 \\
\mathrm{~kg} / \mathrm{m}^{2} \text { ) }\end{array}$ & 2 Recall $24 \mathrm{~h}$ & $56.1 \pm 25.4 \%$ of TEI \\
\hline Rohatgi et al., (2017) [38] & USA & $\begin{array}{l}n=45 \text { pregnant women }(100 \% \mathrm{~W}) \\
\quad \text { (mean age: } \mathrm{nd} \text {; mean BMI: } \mathrm{nd})\end{array}$ & FFQ & $54.4 \pm 13.2 \%$ of TEI \\
\hline Martínez-Steele et al., (2016) [50] & USA & $\begin{array}{c}n=9317 \text { subjects }(\% \mathrm{M} / \mathrm{W}: \mathrm{nd}) \\
\text { (mean age: } \text { nd, } \geq 1 \text { years; mean BMI: nd) }\end{array}$ & 2 Recall $24 \mathrm{~h}$ & $\begin{array}{l}57.9 \% \text { of TEI } \\
\text { SD or SEM: nd }\end{array}$ \\
\hline $\begin{array}{l}\text { Gehring et al., (2020 and 2021) } \\
\qquad[85,86]\end{array}$ & France & $\begin{array}{l}n=21,212 \text { adults }+ \text { older subjects }(73.1 \% \mathrm{~W}) \\
\text { (mean age: } 56.3 \pm 13.8 \text { years; mean BMI: nd) }\end{array}$ & $\begin{array}{c}3 \text { Recall } 24 \mathrm{~h} \\
\text { (every } 6 \text { months) }\end{array}$ & $\begin{array}{l}33.1 \% \text { of TEI } \\
\text { SD or SEM: nd }\end{array}$ \\
\hline Srour et al., (2020) [87] & France & $\begin{array}{l}n=104,707 \text { adults }+ \text { older subjects }(79.2 \% \mathrm{~W}) \\
(\text { mean age: } 42.7 \pm 14.5 \text { years; mean BMI: } \mathrm{nd})\end{array}$ & $\begin{array}{l}3 \text { Recall } 24 \mathrm{~h} \text { via web } \\
\text { (every six months; } 2 \text { days of the } \\
\text { weekend and } 1 \text { of the week) }\end{array}$ & $17.3 \pm 9.8 \%$ of TEI \\
\hline Beslay et al., (2020) [15] & France & $\begin{array}{c}n=110,260 \text { adults }+ \text { older subjects }(78.2 \% \mathrm{~W} \text { ) } \\
\text { (mean age: } 43.1 \pm 14.6 \text { years; mean BMI: } 23.8 \pm 4.6 \\
\mathrm{~kg} / \mathrm{m}^{2} \text { ) }\end{array}$ & $\begin{array}{c}3 \text { Recall } 24 \mathrm{~h} \text { via web } \\
\text { (2 week days and } 1 \text { day of the } \\
\text { weekend; more than } 3500 \text { items) }\end{array}$ & $17.1 \pm 10.3 \%$ of TEI \\
\hline Vasseur et al., (2020) [88] & France & $\begin{array}{c}n=105,832 \text { adults }+ \text { older subjects }(78 \% \mathrm{~W}) \\
\text { mean age: } 43.3 \pm 14.7 \text { years; mean BMI: } 23.9 \\
\left.\mathrm{~kg} / \mathrm{m}^{2}\right)\end{array}$ & $\begin{array}{c}3 \text { Recall } 24 \mathrm{~h} \text { via web } \\
\text { (2 week days and } 1 \text { day of the } \\
\text { weekend; more than } 3300 \text { items) }\end{array}$ & $\begin{array}{l}17 \pm 9 \% \text { of TEI } \\
\text { SD or SEM: nd }\end{array}$ \\
\hline Schnabel et al., (2019) [89] & France & $\begin{array}{l}n=44,551 \text { adults }+ \text { older subjects }(73.1 \% \mathrm{~W}) \\
\text { (mean age: } 56.7 \pm 7.5 \text { years; mean BMI: } \mathrm{nd})\end{array}$ & $\begin{array}{c}3 \text { Recall } 24 \mathrm{~h} \text { via web } \\
\text { (3000 common foods and drinks) }\end{array}$ & $29.1 \pm 10.9 \%$ of TEI \\
\hline Adjibade et al., (2019) [90] & France & $\begin{array}{l}n=26,730 \text { adults }+ \text { older subjects }(76.2 \% \mathrm{~W}) \\
(\text { mean age } 47.2 \pm 14.2 \text { years; mean BMI: } \mathrm{nd})\end{array}$ & $\begin{array}{l}3 \text { Recall } 24 \mathrm{~h} \\
\text { (every } 6 \text { months; } 2 \text { days of the } \\
\text { week and } 1 \text { day of the weekend) }\end{array}$ & $32 \pm 11 \%$ of TEI \\
\hline Schnabel et al., (2018) [91] & France & $\begin{array}{c}n=33,343 \text { adults }+ \text { older subjects }(76.4 \% \mathrm{~W}) \\
(\text { mean age: } 50 \pm 14 \text { years; mean BMI: nd) }\end{array}$ & $\begin{array}{l}3 \text { Recall } 24 \mathrm{~h} \text { via web } \\
\text { (every } 6 \text { months; } 3021 \text { common } \\
\text { foods and drinks) }\end{array}$ & $33 \pm 13.7 \%$ of TEI \\
\hline Rauber et al., (2021a) [36] & UK & $\begin{array}{c}n=542 \text { adolescents (\% M/W: } \mathrm{nd} \text { ) } \\
\text { (mean age: nd; range } 11-18 \text { years, mean BMI: nd) }\end{array}$ & 4-day food diary & $\begin{array}{l}67.8 \% \text { of TEI } \\
\text { SD or SEM: nd }\end{array}$ \\
\hline
\end{tabular}


Table 1. Cont.

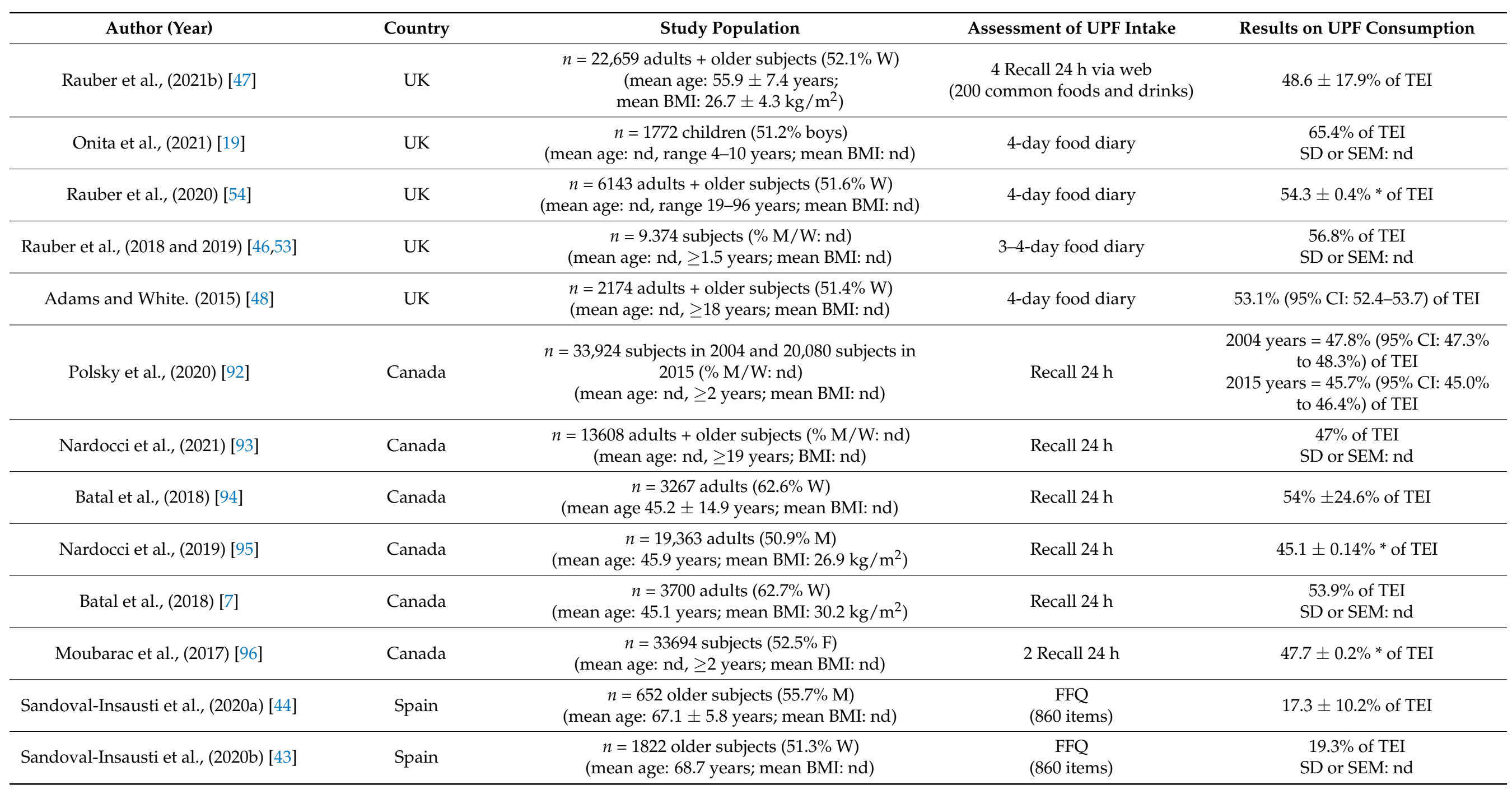


Table 1. Cont.

\begin{tabular}{|c|c|c|c|c|}
\hline Author (Year) & Country & Study Population & Assessment of UPF Intake & Results on UPF Consumption \\
\hline Da Rocha et al., (2020) [20] & Spain & $\begin{array}{c}n=386 \text { children }(52 \% \mathrm{M}) \\
\text { (mean age: } 5.3 \pm 1 \text { years; mean BMI: } 15.7 \pm 1.6 \mathrm{~kg} / \mathrm{m}^{2} \text { ) }\end{array}$ & $\begin{array}{c}\text { FFQ } \\
(149 \text { items })\end{array}$ & $32.2 \pm 8 \%$ of TEI \\
\hline Blanco-Rojo et al., (2019) [97] & Spain & $\begin{array}{c}n=11,898 \text { adults }(50.5 \% \mathrm{~W}) \\
\text { (mean age: } 46.9 \pm 0.3 \text { years; mean BMI: nd) }\end{array}$ & $\begin{array}{c}\text { FFQ } \\
(880 \text { items })\end{array}$ & $24.4 \pm 0.2 \%$ * of TEI \\
\hline Asma' et al., (2020) [98] & Malaysia & $\begin{array}{c}n=200 \text { adults }(75 \% \mathrm{~F}) \\
\text { (mean age: } 33 \text { years; mean BMI: } 25.3 \pm 6.8 \mathrm{~kg} / \mathrm{m}^{2} \text { ) }\end{array}$ & 2 Recall $24 \mathrm{~h}$ & $\begin{array}{l}24 \% \text { of TEI } \\
\text { SD or SEM: nd }\end{array}$ \\
\hline Asma' et al., (2020) [99] & Malaysia & $\begin{array}{c}\qquad n=167 \text { adults }(74.9 \% \mathrm{~F}) \\
\text { (mean age: nd, range } 18-49 \text { years; mean BMI }=24.9 \pm \\
5.2 \mathrm{~kg} / \mathrm{m}^{2} \text { ) }\end{array}$ & $\begin{array}{l}2 \text { Recall } 24 \mathrm{~h} \\
\text { (2 non-consecutive days: } 1 \text { of the } \\
\text { week and } 1 \text { of the weekend) }\end{array}$ & $\begin{array}{l}23 \% \text { of TEI } \\
\text { SD or SEM: nd }\end{array}$ \\
\hline Asma' et al., (2019) [100] & Malaysia & $\begin{array}{c}n=200 \text { adults }(75 \% \mathrm{~F}) \\
\text { (mean age: nd, range } 18-59 \text { years; mean BMI: nd) }\end{array}$ & $\begin{array}{c}\text { FFQ } \\
(165 \text { items })\end{array}$ & $\begin{array}{l}40.4 \% \text { of TEI } \\
\text { SD or SEM: nd }\end{array}$ \\
\hline Machado et al., (2020a) [101] & Australia & $\begin{array}{c}n=7411 \text { adults }+ \text { older subjects }(51.7 \% \mathrm{M}) \\
\text { (mean age: nd, range } 20-85 \text { years; mean BMI: } 27.4 \\
\mathrm{~kg} / \mathrm{m}^{2} \text { ) }\end{array}$ & 2 Recall $24 \mathrm{~h}$ & $\begin{array}{l}38.9 \% \text { of TEI } \\
\text { SD or SEM: nd }\end{array}$ \\
\hline $\begin{array}{l}\text { Machado et al., (2019 and 2020b) } \\
{[102,103]}\end{array}$ & Australia & $\begin{array}{c}n=12153 \text { subjects ( } \% \text { M/W: } \mathrm{nd} \text { ) } \\
\text { (mean age: } \text { nd, } \geq 2 \text { years; mean BMI: nd) }\end{array}$ & 2 Recall $24 \mathrm{~h}$ & $\begin{array}{l}42.0 \% \text { of TEI } \\
\text { SD or SEM: nd }\end{array}$ \\
\hline Vandevijvere et al., (2020) [104] & Belgium & $\begin{array}{c}2004 \text { years, } n=3083 \text { subjects }(\geq 15 \text { years; }(\% \mathrm{M} / \mathrm{W}: \mathrm{nd}) \\
2014-2015 \text { years, } n=3146 \text { subjects (range } 3-64 \text { years; } \\
50.8 \% \mathrm{~F})\end{array}$ & $\begin{array}{c}2 \text { Recall } 24 \mathrm{~h} \text { (adults and } \\
\text { teenagers) } \\
2 \text { food diaries for children (3-9 } \\
\text { years) }\end{array}$ & $\begin{array}{l}\text { Data from } 2004 \text { (survey) }=30.3 \% \\
\quad(95 \% \text { CI: } 29.3-31.5) \text { of TEI } \\
\text { Data from 2014-2015 (survey) }= \\
29.9 \% \text { (95\% CI: } 29.0-30.8) \text { of TEI }\end{array}$ \\
\hline $\begin{array}{l}\text { Khandpur et al., (2020) and Parra } \\
\text { et al., (2019) }[105,106]\end{array}$ & Colombia & $\begin{array}{c}n=38,643 \text { adults }(51.9 \% \mathrm{~F}) \\
\text { (mean age: } 26.5 \pm 0.2 \text { years; mean BMI: nd) }\end{array}$ & Recall $24 \mathrm{~h}$ & $15.9 \pm 0.3 \%$ * of TEI \\
\hline Monge et al., (2020) [107] & Mexico & $\begin{array}{c}n=64934 \text { adults }(100 \% \mathrm{~F}) \\
\text { (mean age: } 41.7 \pm 7.2 \text { years; mean BMI: nd) }\end{array}$ & $\begin{array}{c}\text { FFQ } \\
(140 \text { items) }\end{array}$ & $29.8 \pm 9.4 \%$ of TEI \\
\hline $\begin{array}{l}\text { Marrón-Ponce et al., (2018 and 2019) } \\
\text { [108,109] }\end{array}$ & Mexico & $\begin{array}{c}n=10,087 \text { subjects }(50.5 \% \mathrm{~W}) \\
\text { (mean age: } \mathrm{nd}, \geq 1 \text { years; mean BMI: nd) }\end{array}$ & Recall $24 \mathrm{~h}$ & $30.0 \pm 4.5 \% *$ of TEI \\
\hline Bonaccio et al., (2021) [55] & Italy & $\begin{array}{c}n=24,325 \text { adults }+ \text { older subjects (\% M/W: nd) } \\
\text { (mean age: } n d, \geq 35 \text { years; mean BMI: } n d)\end{array}$ & $\begin{array}{c}\text { FFQ } \\
\text { (188 items) }\end{array}$ & $\begin{array}{l}10 \% \text { of TEI } \\
\text { SD or SEM: nd }\end{array}$ \\
\hline
\end{tabular}


Table 1. Cont.

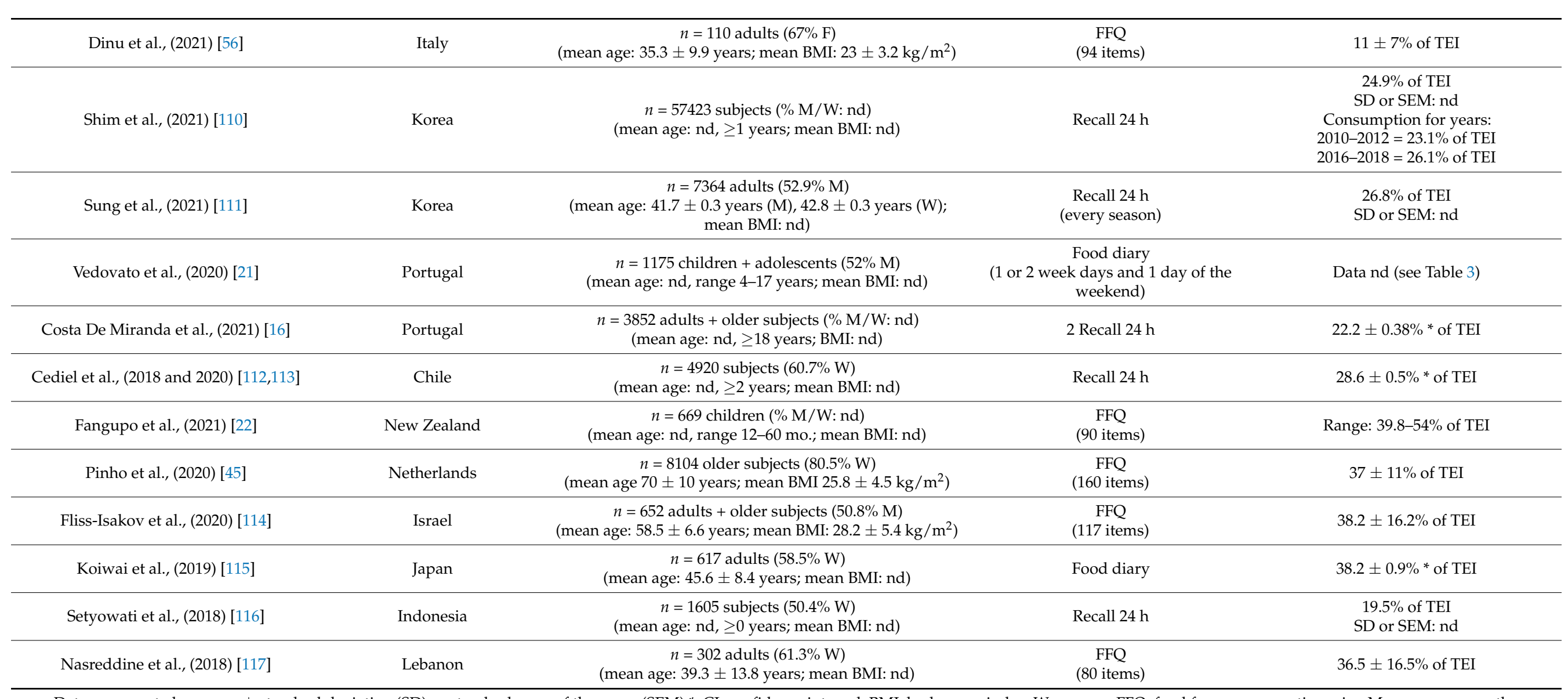

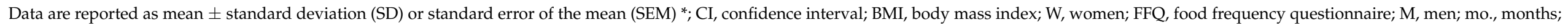
nd, not determined or reported; UPF, ultra-processed food and drink products; TEI, total energy intake. 
Several studies stratified data based on different characteristics, such as age, sex, and BMI. The level of UPF consumption stratified for these parameters is reported in Tables 2-4. Slight differences between the sexes were observed in UPF intake, with men having often an overall higher intake compared to women $[48,54,87,95,96,111]$. However, in most of the studies, the levels of UPF intake appeared comparable [14,30,49,51,81,83,94,112] (Table 2). Regarding age, studies generally reported large variations amongst the age groups. However, UPF intake generally decreased with increasing age, with the highest levels of UPF intake observed in children and adolescents, and the lowest in older subjects $[51,54,87,104,108,110,112,118]$ (Table 3). Only five studies also stratified results based on body mass index (BMI) of the participants, generally finding a slight, but higher, UPF intake in subjects with the highest BMI (e.g., greater than $30 \mathrm{~kg} / \mathrm{m}^{2}$ ) $[58,87,89,95]$ (Table 4) [58].

Table 2. Level of consumption of ultra-processed foods (UPF) expressed as percent energy provided by UPF intake with respect to total energy intake (TEI) considering sex.

\begin{tabular}{|c|c|c|}
\hline Author (Year) & Sex & UPF Consumption and Statistic \\
\hline Adams and White (2015) [48] & $\begin{aligned} \mathrm{F} & =51.4 \% \\
\mathrm{M} & =48.6 \%\end{aligned}$ & $\begin{array}{c}\mathrm{F}=52.8 \%(95 \% \mathrm{CI} 51.9-53.7) \text { of TEI } \\
\mathrm{M}=53.5 \%(95 \% \mathrm{CI} 52.3-54.4) \text { of TEI } \\
\text { Significantly higher }(p<0.05) \text { in men compared } \\
\text { to women }\end{array}$ \\
\hline Baraldi et al., (2018) [49] & (\% M/F: nd) & $\begin{array}{l}\mathrm{F}=58.8 \%(95 \% \mathrm{CI}: 58.1-59.5) \text { of TEI } \\
\mathrm{M}=58.3 \% \text { (95\% CI: } 57.6-59.0) \text { of TEI } \\
\quad \text { No differences between genders }\end{array}$ \\
\hline Batal et al., (2018) [94] & $\begin{array}{l}\mathrm{F}=62.6 \% \\
\mathrm{M}=37.4 \%\end{array}$ & $\begin{array}{c}\mathrm{F}=54.4 \pm 24.4 \% \text { of TEI } \\
\mathrm{M}=53.4 \pm 25.1 \% \text { of TEI } \\
\text { No differences between genders }\end{array}$ \\
\hline Bielemann et al., (2015) [58] & $\begin{aligned} \mathrm{F} & =48.6 \% \\
\mathrm{M} & =51.4 \%\end{aligned}$ & $\begin{array}{c}\mathrm{F}=51.9 \%(95 \% \mathrm{CI}: 51.4-52.5) \text { of TEI } \\
\mathrm{M}=50.4 \%(95 \% \mathrm{CI}: 49.9-51.0) \text { of TEI } \\
\text { Significantly higher }(p<0.001) \text { in women } \\
\text { compared to men }\end{array}$ \\
\hline Calixto Andrade et al., (2021) [84] & $\begin{array}{l}\mathrm{F}=63.3 \% \\
\mathrm{M}=36.7 \%\end{array}$ & $\begin{array}{c}\mathrm{F}=31.4 \% \text { (95\% CI: } 30.1-32.7) \text { of TEI } \\
\mathrm{M}=30.9 \% \text { (95\% CI: } 30.0-31.9) \text { of TEI } \\
\text { Statistics: } \mathrm{nd}\end{array}$ \\
\hline Cediel et al., (2018 and 2020) [112,113] & $\begin{array}{l}\mathrm{F}=60.7 \% \\
\mathrm{M}=39.3 \%\end{array}$ & $\begin{aligned} & \mathrm{F}= 29.4 \%(95 \% \mathrm{CI}: 28.1-30.6) \text { of TEI } \\
&\mathrm{M}=27.8 \% \text { (95\% CI: } 26.5-29.2) \text { of TEI } \\
& \quad \text { No differences between sexes }\end{aligned}$ \\
\hline Da Rocha et al., (2020) [20] & $\begin{array}{l}\mathrm{F}=48 \% \\
\mathrm{M}=52 \%\end{array}$ & $\begin{array}{l}\mathrm{F}=32.0 \% \text { of TEI } \\
\mathrm{M}=32.3 \% \text { of TEI } \\
\text { SD or SEM nd } \\
\text { Statistics: } \text { nd }\end{array}$ \\
\hline Gupta et al., (2020) [81] & $\begin{aligned} \mathrm{F} & =82.1 \% \\
\mathrm{M} & =17.9 \%\end{aligned}$ & $\begin{array}{l}\mathrm{F}=\text { median } 59.9 \% \pm 10.8 \% \text { of TEI } \\
\mathrm{M}=\text { median } 58.4 \% \pm 10.5 \% \text { of TEI } \\
\quad \text { No differences between sexes }\end{array}$ \\
\hline Khandpur et al., (2020) [105] & $\begin{aligned} \mathrm{F} & =51.9 \% \\
\mathrm{M} & =48.1 \%\end{aligned}$ & $\begin{array}{l}\mathrm{F}=16.2 \% \pm 0.2 \% \text { * of TEI } \\
\mathrm{M}=15.5 \% \pm 0.2 \% \text { *f TEI } \\
\text { Significantly higher }(p=0.007) \text { in women } \\
\text { compared to men }\end{array}$ \\
\hline Machado et al., (2020a) [101] & $\begin{aligned} \mathrm{F} & =48.3 \% \\
\mathrm{M} & =51.7 \%\end{aligned}$ & $\begin{array}{c}\mathrm{F}=38.5 \% \text { of TEI } \\
\mathrm{M}=40.7 \% \text { of TEI } \\
\text { SD or SEM nd } \\
\text { No differences between sexes }\end{array}$ \\
\hline $\begin{array}{c}\text { Marrón-Ponce et al., (2018 and 2019) } \\
{\left[\begin{array}{l}108,109]\end{array}\right.}\end{array}$ & $\begin{array}{l}\mathrm{F}=50.5 \% \\
\mathrm{M}=49.5 \%\end{array}$ & $\begin{array}{c}\mathrm{F}=30.1 \% \text { of TEI } \\
\mathrm{M}=29.5 \% \text { of TEI } \\
\text { SD or SEM: } \mathrm{nd} \\
\text { No differences between sexes }\end{array}$ \\
\hline
\end{tabular}


Table 2. Cont.

\begin{tabular}{|c|c|c|}
\hline Author (Year) & Sex & UPF Consumption and Statistic \\
\hline Martínez-Steele et al., (2019) [83] & (\% M/F: nd) & $\begin{array}{c}\mathrm{F}=55.0 \pm 0.5 \% * \text { of TEI } \\
\mathrm{M}=55.9 \pm 0.6 \% \text { * of TEI } \\
\text { No differences between sexes }\end{array}$ \\
\hline Martinez-Steele et al., (2020) [51] & (\% M/F: nd) & $\begin{array}{c}\mathrm{F}=58.2 \pm 0.5 * \% \text { of TEI } \\
\mathrm{M}=58.4 \pm 0.4 * \% \text { of TEI } \\
\text { No differences between sexes }\end{array}$ \\
\hline Moubarac et al., (2017) [96] & $\begin{array}{c}\mathrm{F}=52.5 \% \\
\mathrm{M}=47.5 \%\end{array}$ & $\begin{array}{c}\qquad \mathrm{F}=46.5 \% \text { of TEI } \\
\mathrm{M}=48.6 \% \text { of TEI } \\
\text { SD or SEM nd } \\
\text { Significantly higher }(p<0.001) \text { in men compared } \\
\text { to women }\end{array}$ \\
\hline Nardocci et al., (2019) [95] & $\begin{array}{c}\mathrm{F}=49.1 \% \\
\mathrm{M}=50.9 \%\end{array}$ & $\begin{array}{c}\qquad \mathrm{F}=44.2 \pm 0.4 \% \text { * of TEI } \\
\mathrm{M}=45.9 \pm 0.4 \% \text { * of TEI } \\
\text { Significantly higher }(p<0.05) \text { in men compared } \\
\text { to women }\end{array}$ \\
\hline Rauber et al., (2020) [54] & $\begin{array}{c}\mathrm{F}=51.6 \% \\
\mathrm{M}=48.4 \%\end{array}$ & $\begin{array}{c}\qquad \mathrm{F}=52.8 \pm 0.4 \% \text { * of TEI } \\
\mathrm{M}=55.9 \pm 0.6 \% \text { *f TEI } \\
\text { Significantly higher }(p<0.05) \text { in men compared } \\
\text { to women }\end{array}$ \\
\hline Sandoval-Insausti et al., (2020b) [43] & $\begin{array}{c}\mathrm{F}=51.3 \% \\
\mathrm{M}=48.7 \%\end{array}$ & $\begin{array}{c}\mathrm{F}=20.7 \% \text { of } \mathrm{TEI} \\
\mathrm{M}=17.7 \% \text { of TEI } \\
\text { SD or SEM nd } \\
\text { Statistics: } \text { nd }\end{array}$ \\
\hline Schnabel et al., (2019) [89] & $\begin{array}{c}F=73.1 \% \\
M=26.9 \%\end{array}$ & $\begin{array}{l}\qquad \mathrm{F}=29.4 \pm 0.06 \% \text { * of TEI } \\
\mathrm{M}=28.3 \pm 0.10 \% \text { * of TEI } \\
\text { Significantly higher }(p<0.001) \text { in women } \\
\text { compared to men }\end{array}$ \\
\hline Shim et al., (2021) [110] & (\% M/F: nd) & $\begin{array}{c}\mathrm{F}=24.1 \%(95 \% \mathrm{CI}: 23.8-24.4) \text { of TEI } \\
\mathrm{M}=25.8 \%(95 \% \mathrm{CI}: 25.5-26.1) \text { of } \mathrm{TEI} \\
\text { Significantly higher }(p<0.05) \text { in men compared } \\
\text { to women }\end{array}$ \\
\hline Simões et al., (2018) [73] & $\begin{array}{c}\mathrm{F}=54.2 \% \\
\mathrm{M}=45.8 \%\end{array}$ & $\begin{array}{c}\mathrm{F}=23.0 \%(\mathrm{IQR}: 16.7-29.9) \text { of TEI } \\
\mathrm{M}=20.6 \%(\mathrm{IQR}: 14.7-27.5) \text { of TEI } \\
\text { Significantly higher }(p<0.001) \text { in women } \\
\text { compared to men }\end{array}$ \\
\hline Smiljanec et al., (2020) [14] & $\begin{aligned} \mathrm{F} & =62.5 \% \\
\mathrm{M} & =37.5 \%\end{aligned}$ & $\begin{array}{c}\mathrm{F}=50.8 \pm 2.4 \% \text { of TEI } \\
\mathrm{M}=48.8 \pm 5.2 \% \text { of TEI } \\
\text { No differences between sexes }\end{array}$ \\
\hline Sparrenberger et al., (2015) [30] & $\begin{array}{c}\mathrm{F}=50 \% \\
\mathrm{M}=50 \%\end{array}$ & $\begin{array}{c}\mathrm{F}=47.1 \pm 1.5 \% \text { * of TEI } \\
\mathrm{M}=49.2 \pm 1.6 \% \text { *f TEI } \\
\text { No differences between sexes }\end{array}$ \\
\hline Srour et al., (2020) [87] & $\begin{array}{c}\mathrm{F}=79.2 \% \\
\mathrm{M}=20.8 \%\end{array}$ & $\begin{array}{c}\qquad \mathrm{F}=17.2 \pm 9.7 \% \text { of TEI } \\
\mathrm{M}=17.6 \pm 9.9 \% \text { of TEI } \\
\text { Significantly higher }(p<0.001) \text { in men compared } \\
\text { to women }\end{array}$ \\
\hline Sung et al., (2021) [111] & $\begin{aligned} \mathrm{F} & =47.1 \% \\
\mathrm{M} & =52.9 \%\end{aligned}$ & $\begin{array}{c}\mathrm{F}=25.1 \pm 0.38 \% \text { * of TEI } \\
\mathrm{M}=28.4 \% \pm 0.36 \% \text { * of TEI } \\
\text { Significantly higher }(p<0.0001) \text { in men } \\
\text { compared to women }\end{array}$ \\
\hline
\end{tabular}


Table 2. Cont.

\begin{tabular}{ccc}
\hline Author (Year) & Sex & UPF Consumption and Statistic \\
\hline & & $2004=$ \\
Vandevijvere et al., (2019) [119] & $2004=(\% \mathrm{M} / \mathrm{F}: \mathrm{nd})$ & $\mathrm{F}=28.9 \%(95 \%$ CI: $27.1-30.2)$ of TEI \\
& $2014-2015=$ & $\mathrm{M}=32.3 \%(95 \%$ CI: $30.9-34.3)$ of TEI \\
$2014-2015=$ & $\mathrm{F}=29.7 \%(95 \%$ CI: $28.7-31.2)$ of TEI \\
& $\mathrm{F}=50.8 \%$ & $\mathrm{M}=29.9 \%(95 \%$ CI: $28.6-31.2)$ of TEI \\
Statistics: nd
\end{tabular}

Data are reported as mean \pm standard deviation (SD) or standard error of the mean (SEM) * CI, confidence interval; $\mathrm{F}$, female; $\mathrm{M}$, male; $\mathrm{ND}$, not determined or reported; UPF, ultra-processed food and drink products; TEI, total energy intake.

Table 3. Level of consumption of ultra-processed foods (UPF) expressed as percent energy provided by UPF intake with respect to total energy intake (TEI) by considering age.

\begin{tabular}{|c|c|c|}
\hline Author (Year) & Age & UPF Consumption for Age and Statistics \\
\hline Costa et al., (2021) [60] & $\begin{array}{c}n=3128 \text { children }(6 \text { years }) \\
n=3454 \text { adolescents ( } 11 \text { years) }\end{array}$ & $\begin{array}{c}6 \text { years }=42 \%(\text { IQR: } 34.6-49.8) \text { of TEI } \\
11 \text { years }=32.7 \% \text { (IQR: } 25.1-41.3) \text { of TEI } \\
\text { Statistic: } \text { nd }\end{array}$ \\
\hline Calixto Andrade et al., (2021) [84] & $\begin{array}{c}18-39 \text { years }=34.1 \% \\
40-59 \text { years }=44.8 \% \\
>60 \text { years }=21.1 \%\end{array}$ & $\begin{array}{c}18-39 \text { years }=39.1 \%(95 \% \text { CI: } 37.8-40.5) \text { of TEI } \\
40-59 \text { years }=28.1 \%(95 \% \text { CI: } 27.2-29.0) \text { of TEI } \\
>60 \text { years }=21.6 \%(95 \% \text { CI: } 20.4-22.8) \text { of TEI } \\
\text { Statistics: } \text { nd }\end{array}$ \\
\hline Shim et al., (2021) [110] & nd & $\begin{array}{c}1-12 \text { years }=28.9 \%(95 \% \text { CI: } 28.5-29.4) \text { of TEI } \\
13-19 \text { years }=32.6 \%(95 \% \text { CI: } 31.9-33.4) \text { of TEI } \\
20-49 \text { years }=27.7 \%(95 \% \text { CI: } 27.3-28.0) \text { of TEI } \\
50-64 \text { years }=19.6 \%(95 \% \text { CI: } 19.2-19.9) \text { of TEI } \\
>65 \text { years }=15.1 \%(95 \% \text { CI: } 14.8-15.8) \text { of TEI } \\
\text { Significantly higher }(p<0.05) \text { in adolescents and } \\
\text { lower in subjects older than } 65 \text { years }\end{array}$ \\
\hline Sung et al., (2021) [111] & $\begin{array}{l}n=1114(19-29 \text { years }) \\
n=3301(30-49 \text { years }) \\
n=2949(50-64 \text { years })\end{array}$ & $\begin{array}{c}\text { 19-29 years }=35.7 \pm 0.6 \% \text { * of TEI } \\
30-49 \text { years }=27.7 \pm 0.4 \% \text { * of TEI } \\
50-64 \text { years }=20.0 \pm 0.4 \% \text { * of TEI } \\
\text { Significantly higher }(p<0.0001) \text { in younger }\end{array}$ \\
\hline Costa De Miranda et al., (2021) [16] & $\begin{array}{c}n=3102(18-65 \text { years }) \\
n=750(>65 \text { years })\end{array}$ & $\begin{array}{c}\text { 18-65 years }=23.8 \pm 0.42 \% \text { * of TEI } \\
>65 \text { years }=15.9 \pm 0.56 \% \text { * of TEI } \\
\text { Significant differences }(p=0.001) \text { between adults } \\
\text { and older subjects }\end{array}$ \\
\hline Fangupo et al., (2021) [22] & $\begin{array}{l}n=501(12 \mathrm{mo}) \\
n=497(24 \mathrm{mo}) \\
n=475(36 \mathrm{mo})\end{array}$ & $\begin{array}{c}12 \mathrm{mo}=44.5 \%(95 \% \text { CI: } 43.0-46.0) \text { of TEI } \\
24 \mathrm{mo}=39.8 \%(95 \% \mathrm{CI}: 34.6-41.0) \text { of TEI } \\
60 \mathrm{mo}=54 \%(95 \% \mathrm{CI}: 53.0-54.9) \text { of TEI } \\
\text { Intraclass correlation coefficients ranging from } \\
0.23 \text { to } 0.36\end{array}$ \\
\hline Leffa et al., (2020) [24] & $n=308$ children ( 3 and 6 years) & $\begin{array}{c}3 \text { years }=43.4 \%(\text { IQR } 34.3-51.1 \%) \text { of TEI } \\
6 \text { years }=47.7 \%(\text { IQR } 41.5-53.8 \%) \text { of TEI } \\
\text { Significant differences }(p<0.001) \text { between age }\end{array}$ \\
\hline Gupta et al., (2020) [81] & $\begin{array}{c}n=286(21-40 \text { years }) \\
n=230(41-50 \text { years }) \\
n=239(\geq 51 \text { years })\end{array}$ & $\begin{array}{c}21-40 \text { years }=60.2 \pm 11.1 \% \text { of TEI } \\
41-50 \text { years }=60.6 \pm 10.0 \% \text { of TEI } \\
\geq 51 \text { years }=58.1 \pm 10.9 \% \text { of TEI } \\
\text { Consumption at } \geq 51 \text { years, but not at } 41-50 \\
\text { years, significantly lower }(p<0.05) \text { compared to } \\
21-40 \text { years }\end{array}$ \\
\hline
\end{tabular}


Table 3. Cont.

\begin{tabular}{|c|c|c|}
\hline Author (Year) & Age & UPF Consumption for Age and Statistics \\
\hline Martinez Steele et al., (2020) [51] & nd & $\begin{array}{c}\text { 6-11 years }=68.2 \pm 0.5 \% \text { * of TEI } \\
12-19 \text { years }=66.9 \pm 0.7 \% \text { * of TEI } \\
>20 \text { years }=55.9 \pm 0.4 \% \text { * of TEI } \\
\text { Significantly lowest }(p<0.05) \text { at }>20 \text { years }\end{array}$ \\
\hline Srour et al., (2020) [87] & $\begin{array}{c}n=59,247(18-44 \text { years }) \\
n=28,930(45-59 \text { years }) \\
n=16,530(>60 \text { years })\end{array}$ & $\begin{array}{c}\text { 18-44 years }=19.4 \pm 10.6 \% \text { of TEI } \\
45-59 \text { years }=14.7 \pm 8 \% \text { of TEI } \\
>60 \text { years }=14 \pm 7.2 \% \text { of TEI } \\
\text { Significant differences }(p<0.001) \text { between groups }\end{array}$ \\
\hline Rauber et al., (2020) [54] & $\begin{array}{c}19-29 \text { years }=18.7 \% \\
30-59 \text { years }=51.0 \% \\
>60 \text { years }=30.3 \%\end{array}$ & $\begin{array}{c}\text { 19-29 years }=59.2 \pm 1.3 \% \text { * of TEI } \\
30-59 \text { years }=54 \pm 0.4 \% \text { * of TEI } \\
>60 \text { years }=51.8 \pm 0.5 \% \text { * of TEI } \\
\text { Significant differences }(p<0.05) \text { in the group of subjects } \\
\text { aged }>60 \text { years }\end{array}$ \\
\hline Polsky et al., (2020) [92] & nd & 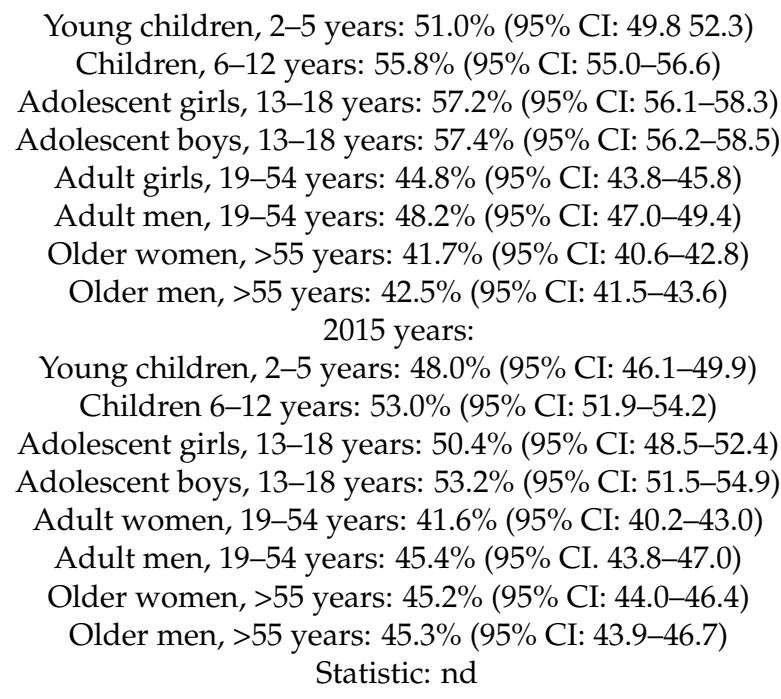 \\
\hline Machado et al., (2020a) [101] & $\begin{array}{c}20-39 \text { years }=38.5 \% \\
40-59 \text { years }=36.4 \% \\
>60 \text { years }=25.1 \%\end{array}$ & $\begin{array}{l}20-39 \text { years }=43.4 \% \text { of TEI } \\
40-59 \text { years }=36.2 \% \text { of TEI } \\
>60 \text { years }=36.2 \% \text { of TEI } \\
\text { SD or SE: } \text { nd } \\
\text { Statistics: } \text { nd }\end{array}$ \\
\hline Khandpur et al., (2020) [105] & $\begin{array}{c}2-9 \text { years }=18.5 \% \\
10-19 \text { years }=23.0 \% \\
20-34 \text { years }=26.3 \% \\
35-49 \text { years }=20.0 \% \\
\geq 50 \text { years }=12.2 \%\end{array}$ & $\begin{array}{c}\text { 2-9 years }=19.3 \pm 0.3 \% \text { * of TEI } \\
10-19 \text { years }=19.3 \pm 0.3 \% \text { * of TEI } \\
20-34 \text { years }=15.4 \pm 0.3 \% \text { * of TEI } \\
35-49 \text { years }=12.2 \pm 0.3 \% \text { * of TEI } \\
\geq 50 \text { years }=11.4 \pm 0.4 \% \text { * of TEI } \\
\text { Significant differences }(p<0.001) \text { between age groups }\end{array}$ \\
\hline Vedovato et al., (2020) [21] & $n=1175$ children ( 3 and 7 years) & $\begin{array}{c}4 \text { years }=27.3 \pm 11.1 \% \text { of TEI } \\
7 \text { years }=29.3 \pm 10.4 \% \text { of TEI } \\
\text { Interclass correlation coefficient }=0.32\end{array}$ \\
\hline $\begin{array}{c}\text { Machado et al., (2019 and 2020b) } \\
{[102,103]}\end{array}$ & $\begin{array}{c}n=822(2-5 \text { years }) \\
n=889(6-11 \text { years }) \\
n=1204(12-19 \text { years }) \\
n=7135(20-64 \text { years }) \\
n=2103(>65 \text { years })\end{array}$ & $\begin{array}{c}2-5 \text { years }=47.3 \% \text { of TEI } \\
6-11 \text { years }=53.1 \% \text { of TEI } \\
12-19 \text { years }=54.3 \% \text { of TEI } \\
20-64 \text { years }=39.4 \% \text { of TEI } \\
>65 \text { years }=36.3 \% \text { of TEI } \\
\text { SD or SE: } \text { nd } \\
\text { Statistic: } \text { nd }\end{array}$ \\
\hline
\end{tabular}


Table 3. Cont.

\begin{tabular}{|c|c|c|}
\hline Author (Year) & Age & UPF Consumption for Age and Statistics \\
\hline $\begin{array}{l}\text { Vandevijvere et al., (2019) and } \\
\qquad(2020)[104,119]\end{array}$ & $\begin{array}{c}n=992(3-9 \text { years }) \\
n=928(10-17 \text { years }) \\
n=1226(18-64 \text { years })\end{array}$ & $\begin{array}{c}\text { 3-9 years }=33.3 \%(95 \% \text { CI: } 32.1-35.0) \text { of TEI } \\
10-17 \text { years }=29.2 \%(95 \% \text { CI: } 27.7-30.3) \text { of TEI } \\
\text { 18-64 years }=29.6 \%(28.5-30.7) \text { of TEI } \\
\text { Significantly higher }(p<0.05) \text { in children compared to } \\
\text { adolescents and adults }\end{array}$ \\
\hline Neri et al., (2019) [18] & $\begin{array}{c}n=2411(2-5 \text { years }) \\
n=3335(6-11 \text { years }) \\
n=3726(12-19 \text { years })\end{array}$ & $\begin{array}{l}2-5 \text { years }=58.2 \% \text { of TEI } \\
6-11 \text { years }=66.2 \% \text { of TEI } \\
12-19 \text { years }=66.4 \% \text { of TEI } \\
\text { SD or SE: } \text { nd } \\
\text { Statistics: } \text { nd }\end{array}$ \\
\hline Martínez-Steele et al., (2019) [83] & $\begin{array}{c}n=2126(20-39 \text { years }) \\
n=2239(40-59 \text { years }) \\
n=2020(>60 \text { years })\end{array}$ & $\begin{array}{c}\text { 20-39 years }=58.9 \pm 0.6 \% \text { * of TEI } \\
40-59 \text { years }=54.6 \pm 0.8 \% \text { * of TEI } \\
>60 \text { years }=52.2 \pm 0.6 \% \text { * of TEI } \\
\text { Significantly lower }(p<0.001) \text { at }>60 \text { years compared to } \\
40-59 \text { and } 20-39 \text { years }\end{array}$ \\
\hline Schnabel et al., (2019) [89] & nd & $\begin{array}{c}\text { 45-64 years }=29.6 \pm 0.06 \% \text { * of TEI } \\
\geq 65 \text { years }=26.3 \pm 0.13 \% \text { * of TEI } \\
\text { Significant differences }(p<0.001) \text { between groups }\end{array}$ \\
\hline Nardocci et al., (2019) [95] & nd & $\begin{array}{c}\text { 18-34 years }=50.2 \pm 0.55 \% \text { * of TEI } \\
\text { 35-44 years }=20.6 \pm 0.77 \% \text { * of TEI } \\
45-64 \text { years }=34.9 \% \pm 0.46 \% \text { * of TEI } \\
>65 \text { years }=41.9 \pm 0.42 \% \text { * of TEI } \\
\text { Significantly lower }(p<0.05) \text { at } 35-55 \text { years, } 45-64 \text { years, } \\
\text { and }>65 \text { years compared to } 18-34 \text { years }\end{array}$ \\
\hline $\begin{array}{l}\text { Cediel et al., (2018 and 2020) } \\
{\left[\begin{array}{c}112,113]\end{array}\right.}\end{array}$ & $\begin{array}{c}n=1374(2-19 \text { years }) \\
n=1668(20-49 \text { years }) \\
n=948(50-64 \text { years }) \\
n=930(>65 \text { years })\end{array}$ & $\begin{array}{c}2-19 \text { years }=38.6 \%(95 \% \text { CI: } 35.7-39.4) \text { of TEI } \\
20-49 \text { years }=26.7 \%(95 \% \text { CI: } 26.2-29.1) \text { of TEI } \\
50-64 \text { years }=21.8 \%(95 \% \text { CI: } 19.5-23.6) \text { of TEI } \\
>65 \text { years }=18.3 \%(95 \% \text { CI: } 15.9-18.9) \text { of TEI } \\
\text { Significantly lower }(p<0.001) \text { at }>65 \text { years }\end{array}$ \\
\hline $\begin{array}{l}\text { Marrón-Ponce et al., (2018 and } \\
\text { 2019) }[108,109]\end{array}$ & $\begin{array}{c}1-4 \text { years }=7.6 \% \\
5-11 \text { years }=16.1 \% \\
12-19 \text { years }=14.5 \% \\
>20 \text { years }=61.8 \%\end{array}$ & $\begin{array}{l}1-4 \text { years }=38.6 \% \text { of TEI } \\
5-11 \text { years }=34.3 \% \text { of TEI } \\
12-19 \text { years }=35.5 \% \text { of TEI } \\
\geq 20 \text { years }=26.2 \% \text { of TEI } \\
\text { SD or SEM: } n d\end{array}$ \\
\hline
\end{tabular}

Significant differences $(p<0.05)$ between age groups

Rauber et al., (2018 and 2019) $[46,53]$

nd
$1.5-10$ years $=63.5 \pm 0.34 \%$ * of TEI

$11-18$ years $=68.0 \pm 0.40 \%$ * of TEI

$19-64$ years $=54.9 \pm 0.35 \%$ * of TEI

$>65$ years $=53.0 \pm 0.52 \%$ * of TEI

Significant differences $(p<0.001)$ in children and adolescents
$0-4$ years $=6.5 \%$

$5-12$ years $=14.1 \%$

Setyowati et al., (2018) [116]
$13-18$ years $=11.5 \%$

$19-55$ years $=55 \%$

$>55$ years $=12.9 \%$

$$
\begin{gathered}
0-4 \text { years }=41.4 \% \text { of TEI } \\
5-12 \text { years }=29 \% \text { of TEI } \\
13-18 \text { years }=27.8 \% \text { of TEI } \\
19-55 \text { years }=16.1 \% \text { of TEI } \\
>55 \text { years }=9 \% \text { of TEI } \\
\text { SD or SE: } \text { nd } \\
\text { Statistic: } \text { nd }
\end{gathered}
$$

$35-44$ years $=24.8 \%($ IQR: $18.6-31.5)$ of TEI

$45-54$ years $=22.2 \%$ (IQR $16.3-29.0)$ of TEI

$55-64$ years $=20 \%$ (IQR 14.1-26.7) of TEI

$65-74$ years $=19.5 \%($ IQR $13.5-26.4)$ of TEI

Significantly higher $(p<0.001)$ in the group aged 35-44 years and then decreasing with age 
Table 3. Cont.

\begin{tabular}{|c|c|c|}
\hline Author (Year) & Age & UPF Consumption for Age and Statistics \\
\hline Baraldi et al., (2018) [49] & nd & $\begin{array}{c}2-9 \text { years }=65.9 \%(95 \% \text { CI: } 65.0-66.8) \text { of TEI } \\
10-19 \text { years }=66.8 \%(95 \% \text { CI: } 65.9-67.7) \text { of TEI } \\
20-39 \text { years }=59.5 \%(95 \% \text { CI: } 58.7-60.3) \text { of TEI } \\
40-59 \text { years }=55.2 \%(95 \% \text { CI: } 54.1-56.4) \text { of TEI } \\
>60 \text { years }=52.8 \%(95 \% \text { CI: } 51.9-53.7) \text { of TEI } \\
\text { Significantly lowest }(p<0.05) \text { at }>60 \text { years }\end{array}$ \\
\hline Moubarac et al., (2017) [96] & $\begin{array}{c}n=13,779(2-18 \text { years }) \\
n=3812(19-30 \text { years }) \\
n=5601 \text { ( } 31-50 \text { years }) \\
n=4611(51-64 \text { years }) \\
n=5891(>65 \text { years })\end{array}$ & $\begin{array}{c}2-18 \text { years }=55.1 \% \text { of TEI } \\
19-30 \text { years }=51 \% \text { of TEI } \\
31-50 \text { years }=44.9 \% \text { of TEI } \\
51-64 \text { years }=42.4 \% \text { of TEI } \\
>65 \text { years }=42.6 \% \text { of TEI } \\
\text { SD or SE: } \text { nd } \\
\text { Significantly higher }(p<0.001) \text { at }>65 \text { years }\end{array}$ \\
\hline Karnopp et al., (2017) [29] & $\begin{array}{l}<24 \mathrm{mo}=72.5 \% \\
>24 \mathrm{mo}=27.5 \%\end{array}$ & $\begin{array}{c}<24 \mathrm{mo}=19.7 \pm 1.3 \% * \text { of TEI } \\
>24 \mathrm{mo}=36 \pm 0.8 \% * \text { of TEI } \\
\text { Statistics: } \mathrm{nd}\end{array}$ \\
\hline Sparrenberger et al., (2015) [30] & $\begin{array}{c}n=66(55 \% \text { F })(2-6 \text { years }) \\
n=36(43.4 \% \text { F })(7-10 \text { years })\end{array}$ & $\begin{array}{c}2-6 \text { years }=43.7 \pm 1.4 \% \text { * of TEI } \\
7-10 \text { years }=54.7 \pm 1.7 \% \text { * of TEI } \\
\text { Significant differences }(p<0.001) \text { between } \\
\text { groups }\end{array}$ \\
\hline Adams and White (2015) [48] & $\begin{array}{c}18-29 \text { years }=19.3 \% \\
30-39 \text { years }=17.0 \% \\
40-49 \text { years }=19.0 \% \\
50-59 \text { years }=15.7 \% \\
60-69 \text { years }=13.8 \% \\
\geq 70 \text { years }=15.2 \%\end{array}$ & $\begin{array}{c}18-29 \text { years }=58.2 \%(95 \% \text { CI: } 56.3-60.2) \text { of TEI } \\
30-39 \text { years }=55.9 \%(95 \% \text { CI: } 54.5-57.3) \text { of TEI } \\
40-49 \text { years }=52.5 \%(95 \% \text { CI: } 50.7-53.6) \text { of TEI } \\
50-59 \text { years }=49.7 \%(95 \% \text { CI: } 48.1-51.3) \text { of TEI } \\
60-69 \text { years }=49 \%(95 \% \text { CI: } 47.5-50.5) \text { of TEI } \\
\geq 70 \text { years }=50.6 \%(95 \% \text { CI: } 49.0-52.2) \text { of TEI } \\
\text { Significant negative association between age and } \\
\text { percentage of TEI from UPF }\end{array}$ \\
\hline
\end{tabular}

Data are reported as mean \pm standard deviation (SD) or standard error of the mean (SEM) *; CI, confidence interval; mo, months; ND, not determined or reported; UPF, ultra-processed food and drink products; TEI, total energy intake.

Table 4. Level of consumption of ultra-processed foods (UPF) expressed as \% energy provided by UPF intake with respect to total energy intake (TEI) by considering the BMI factor.

\begin{tabular}{|c|c|c|}
\hline Author (Year) & Body Mass Index (BMI) & UPF Consumption for BMI and Statistics \\
\hline Bielemann et al., (2015) [58] & $\begin{array}{c}\mathrm{BMI}<24.9 \mathrm{~kg} / \mathrm{m}^{2}=70.8 \% \\
\mathrm{BMI} 25-29.9 \mathrm{~kg} / \mathrm{m}^{2}=20.8 \% \\
\mathrm{BMI} \geq 30 \mathrm{~kg} / \mathrm{m}^{2}=8.4 \%\end{array}$ & $\begin{array}{c}\text { BMI } 25-29.9 \mathrm{~kg} / \mathrm{m}^{2}=50.5 \% \text { of TEI } \\
\text { BMI }<24.9 \mathrm{~kg} / \mathrm{m}^{2}=51.6 \% \text { of TEI } \\
\text { SD or SEM nd } \\
\text { Significant differences ( } p=0.003 \text { ) between groups }\end{array}$ \\
\hline Nardocci et al., (2019) [95] & $\begin{array}{c}\text { BMI } 18.5-24.9 \mathrm{~kg} / \mathrm{m}^{2}=40.2 \% \\
\text { BMI } 25-29.9 \mathrm{~kg} / \mathrm{m}^{2}=37.6 \% \\
\text { BMI } \geq 30 \mathrm{~kg} / \mathrm{m}^{2}=22.2 \%\end{array}$ & $\begin{array}{l}\text { BMI } 18.5-24.9 \mathrm{~kg} / \mathrm{m}^{2}=44.3 \pm 0.4 \% \text { * of TEI } \\
\text { BMI } 25-29.9 \mathrm{~kg} / \mathrm{m}^{2}=44.8 \pm 0.45 \% \text { * of TEI } \\
\text { BMI } \geq 30 \mathrm{~kg} / \mathrm{m}^{2}=46.8 \pm 0.6 \% \text { * of TEI } \\
\text { Significantly higher }(p<0.05) \text { in obese subjects }\end{array}$ \\
\hline Schnabel et al., (2019) [89] & nd & $\begin{array}{c}\text { BMI }<18.5 \mathrm{~kg} / \mathrm{m}^{2}=28.3 \pm 0.30 \% \text { * of TEI } \\
\text { BMI } 18.5-24.9 \mathrm{~kg} / \mathrm{m}^{2}=28.6 \pm 0.07 \% \text { * of TEI } \\
\text { BMI } 25-29.9 \mathrm{~kg} / \mathrm{m}^{2}=29.3 \pm 0.10 \% \text { * of TEI } \\
\text { BMI } \geq 30 \mathrm{~kg} / \mathrm{m}^{2}=31.3 \pm 0.16 \% \text { * of TEI } \\
\text { Significant differences }(p<0.001) \text { between groups }\end{array}$ \\
\hline Srour et al., (2020) [87] & $\begin{array}{c}\text { BMI }<25 \mathrm{~kg} / \mathrm{m}^{2}=69.1 \% \\
\text { BMI } 25-29.9 \mathrm{~kg} / \mathrm{m}^{2}=20.2 \% \\
\text { BMI } \geq 30 \mathrm{~kg} / \mathrm{m}^{2}=7.8 \%\end{array}$ & $\begin{array}{c}\text { BMI }<25 \mathrm{~kg} / \mathrm{m}^{2}=17.1 \pm 9.7 \% \text { of TEI } \\
\text { BMI } 25-29.9 \mathrm{~kg} / \mathrm{m}^{2}=17 \pm 9.6 \% \text { of TEI } \\
\text { BMI } \geq 30 \mathrm{~kg} / \mathrm{m}^{2}=18.8 \pm 11.1 \% \text { of TEI } \\
\text { Significant differences }(p<0.001) \text { between groups }\end{array}$ \\
\hline Vandevijvere et al., (2019) [119] & $\begin{array}{c}\text { BMI } 18.5-24.9 \mathrm{~kg} / \mathrm{m}^{2}=40.2 \% \\
\text { BMI } 25-29.9 \mathrm{~kg} / \mathrm{m}^{2}=37.6 \% \\
\text { BMI } \geq 30 \mathrm{~kg} / \mathrm{m}^{2}=22.2 \%\end{array}$ & $\begin{array}{c}\left.\text { BMI 18.5-24.9 kg/m } / \mathrm{m}^{2}=30.7 \% \text { (95\% CI: } 29.1-31.9\right) \text { of TEI } \\
\left.\text { BMI } 25-29.9 \mathrm{~kg} / \mathrm{m}^{2}=28.5 \% \text { (95\% CI: } 27.5-31.1\right) \text { of TEI } \\
\left.\text { BMI } \geq 30 \mathrm{~kg} / \mathrm{m}^{2}=29.3 \% \text { (95\% CI: } 26.6-31.1 \%\right) \text { of TEI } \\
\text { No significant differences between groups }\end{array}$ \\
\hline
\end{tabular}

Data are reported as mean \pm standard deviation $(\mathrm{SD})$ or standard error of the mean (SEM) * CI, confidence interval; BMI, body mass index; ND, not determined or reported; UPF, ultra-processed food and drink products; TEI, total energy intake. 
Two studies evaluated the intake of UPF during pregnancy, reporting conflicting results. Silva et al. [37] reported no difference between second and third trimester. Conversely, Gomes et al. [34] reported a significant difference in the second and third trimesters between the intervention and control group. This latter consumed more UPF compared to the intervention group that received training for the application of healthy food practices during prenatal care appointments (see Supplementary Table S1). Finally, Gehring and colleagues $[85,86]$ compared UPF intake in subjects adhering to different dietary patterns, observing a higher UPF intake in vegans and vegetarians than pesco-vegetarians and meat eaters (Supplementary Table S1).

\section{Discussion}

Since Monteiro et al. proposed the NOVA classification to categorize foods based on the degree of processing [4], several studies have been conducted to estimate the level of consumption of UPF and its association with several health markers $[24,35,52,67,120]$ as well as with disease risk and mortality [55,59,87,121,122], adjusting the models for energy intake and other potential confounding factors. It was hypothesized that a high level of UPF consumption may represent a health issue, being associated with weight gain and worsening of cardiovascular risk factors such as high waist circumference and low HDL cholesterol [11].

In the present study, we collected 100 unique studies published in 106 manuscripts estimating the UPF levels in different populations from 21 countries around the world. Overall, we found a large variability in the percent of TEI obtained from UPF in the different countries, with the United States and United Kingdom being the countries with the highest percent of TEI from UPF, and Mediterranean countries such as Italy showing the lowest level ( $10 \%$ of TEI). These results are in line with previous evidence suggesting that adherence to the Mediterranean diet is inversely associated with UPF consumption [20]. This is further confirmed by findings showing that the highest tertiles or quartiles of UPF intake are associated with the lowest adherence to the Mediterranean diet [44,55]. The low levels of UPF consumption registered in Italy and other Mediterranean countries are those associated with the lowest risks for non-communicable diseases. For instance, da Silva et al. recently observed an association between UPF consumption and the increased presence of high waist circumference, overweight, and peripheral arterial disease when comparing the third and first tertiles of the UPF contribution to energy intake in a Brazilian cohort [123]. Intriguingly, the first tertile corresponded to $<10.6 \%$ of TEI from UPF, which is similar to the levels registered in the study conducted in the Italian population. These levels are far lower than those detected in the first quartiles in the study by Rauber et al., who observed that participants in the highest quartile $(>70.3 \%$ and $>71.7 \%$ of TEI from UPF in women and men, respectively) had a significantly higher risk of developing obesity, and of experiencing a $\geq 5 \%$ increase in $\mathrm{BMI}$ waist circumference than those belonging to the lowest quartile ( $<24.1 \%$ and $<26.3 \%$ of TEI from UPF in women and men, respectively) [47]. The type of foods contributing to UPF intake largely varied among countries, but, in accordance with previous findings [124], the most consumed UPF included: baked goods, dairy products, processed fruit and vegetables, and, among drinks, carbonated drinks.

In addition to country, the level of UPF intake was found to be inversely associated with the increase in age. In this regard, children generally showed the highest intake of UPF, which led the European Childhood Obesity Group to "a call to action" aimed to inform people about the potential harmful effects of UPF [118]. For example, it was found that in the United Kingdom, $65 \%$ of calories eaten by primary and secondary school children derived from white bread, biscuits, carbonated drinks, crisps, and chips. These findings are in line with the observations reported by others $[125,126]$. Similarly, the pediatric populations of the United States and Canada reported an intake of UPF above 55\% by including breads, cookies, savory snacks, reconstituted meat products, milk-based drinks, breakfast cereals, juices and sodas, and frozen and ready-to-eat meals in the diet $[18,96]$. Among U.S. school-aged children and adolescents, UPF provided $66.2 \%$ and $66.4 \%$ of 
TEI, respectively, with pizzas, sodas, and juices being the most-consumed products [118]. In this scenario, it was proposed that the levels of UPF intake in the young may reflect, at least in part, their lifestyle. In this regard, a recent study documented that subjects consume more UPF when dining out than when eating at home [127]. Other reasons are related to socio-economic inequalities, including lower education status of the mother or unemployed parents, which may lead to a preference for cheaper and less nutritious foods [105]. A different trend was observed for older subjects who showed a lower intake of UPF compared to younger subjects; the main UPF products included cookies and pastries, but also processed breads, breakfast cereals, and yogurts [16,128]. Compared to age, a minor variability was found for sex and/or BMI, which might differ for the net amount of UPF consumption but not for the percent of TEI from UPF. Intriguingly, the adherence to specific dietary patterns represented an additional determinant of the levels of UPF consumption. In this context, based on the NOVA classification, vegans and vegetarians reported higher UPF consumption compared to pesco-vegetarians and meat eaters, mainly driven by a higher consumption of plant-based meat and dairy substitutes. These results highlight the high variability in the characteristics of these types of diet, which may differ widely for the consumption of several food groups [129]. However, these results were found only in two different publications belonging to the same cross-sectional trial performed in the NutriNet-Santé cohort [85,86]; thus, a confirmation of such an analysis deserves further investigation, also to comprehend better if all vegetarian diets have the same health benefits regardless of the levels of UPF consumed.

A thorough comparison of the findings from the different studies considered was challenging due to the differences in food classification and to the disparate definitions that were proposed. Descriptions of UPF within the NOVA system vary with distinguishing features including single vs. $2-3$ vs. $\geq 5$ more ingredients, or natural/fresh vs. imitation or industrial, and whole foods vs. fractioned substances [13]. This means that different studies may have classified the same food as UPF or not based on the distinguishing feature used for classifying foods.

Notably, one of the main sources of variation among study protocols is the tools used for estimating UPF intake. Overall, from the present review, it is difficult to provide conclusive findings about the influence of the tools used to determine UPF intake since its estimation was typically performed using a single method. In this regard, most of the studies $(n=49)$ used the $24 \mathrm{~h}$ recall. To reflect the typical diet, this tool needs to be administered several times; however, in some studies, data were derived from a single $24 \mathrm{~h}$ recall, which could have affected the significance of the findings. However, this tool has the strength of being able to assess the consumption of all food items since subjects can include/report specific information (e.g., brand), which may help with identifying the NOVA group. However, a lower number of studies assessed UPF intake using FFQs, which are not always specifically created and validated to estimate the consumption of products undergoing different food processing, and the food list cannot cover all the food items consumed, thus leading to underreporting [130]. Among studies using FFQs, some of them used the questionnaire developed within the European Prospective Investigation into Cancer and Nutrition (EPIC) [131], which is not able to distinguish among products belonging to different NOVA groups. This is, for instance, the case for artisanal or industrial breads or cakes that belong to two different NOVA groups (Groups 3 and 4, respectively). Therefore, the use of tools not specifically validated for estimating UPF consumption may potentially lead to the misclassification of foods in the UPF categories, which, in turn, may lead to the misinterpretation of the associations found with markers of health. Only two studies $[17,51]$ used a questionnaire specifically validated for estimating the levels of UPF consumption in children and adults. The approach to validating and using ad hoc developed FFQs, when possible, created for specific populations to consider the different dietary habits, should be recommended for more accurately estimating the consumption of UPF and the actual impact on health-related outcomes. 
This study has some strengths worth highlighting, the first of which is the rigorous search and selection strategy that identified available studies examining the energy intake from UPF. However, we cannot exclude that the use of further databases may have allowed the identification of additional studies. Secondly, food processing level was always determined according to the criteria of NOVA classification, to facilitate the comparison amongst findings, although it was reported that some NOVA definitions are open to researchers' interpretation [132], leading to a not-always-uniform categorization [133]. Finally, we included only studies reporting results as the percent of TEI from UPF and not as grams per day. In our opinion, the ratio of energy intake from UPF compared to total energy intake is more useful for reflecting the impact of these products on the whole diet. However, this method may not be useful for detecting the consumption of energy-free products (such as energy-free drinks with artificial sweeteners); thus, this choice can also be considered as a limitation. Notably, the NOVA system used has been largely criticized for different reasons, mostly because this system focuses on the role of food processing, regardless of the nutritional characteristics of foods, on human health [132]. Thus, further efforts should be directed toward elucidating the impact of the different foods belonging to the same NOVA category but with different nutrient profiles on human health. To conclude, it seems worthwhile to implement a critical and constructive discussion able to clarify the potential and applicability of this type of approach.

\section{Conclusions}

In conclusion, this review showed high levels of UPF consumption, especially in some countries and in specific target groups (i.e., children and adolescents). However, most of the data on UPF consumption have been derived from FFQs and $24 \mathrm{~h}$ dietary recall, which are not specifically validated for estimating UPF; thus, such data should be considered with caution. In this scenario, tools specifically validated to estimate the levels of UPF consumption can be useful to avoid misinterpretation of the findings, especially when used to investigate the association with health status. Despite several studies reporting a positive association between UPF and obesity and cardiometabolic health, on the whole, the evidence is not yet totally convincing. In addition, whether this association is dependent on the nutritional characteristics of UPF and/or related to the applied processing is unclear. In this context, despite the NOVA system classifying foods based on the food processing technology without providing any information about the nutritional content of the food, the UPF group has been suggested to be an indicator of poor food quality due to the generally high amounts of free or added sugars, fats, low levels of fiber, and high energy density. For instance, it was recently observed that UPF consumption is associated with a deterioration in diet quality, with UPF intake being negatively correlated with fiber and protein and positively correlated with sugar, fat, and saturated fat intake [115]. This highlights that the concept of UPF may be somehow misleading, with the effect on human health mediated more by the nutritional quality of products rather than the processing. The association between UPF and nutritional adequacy is not surprising, since, for instance, the presence of added sugar or fat is a major element in defining UPF. Finally, the classification systems based on processing are not often aligned with dietary guidelines (e.g., some products considered UPF are recommended within a balanced diet). Thus, confirmation of the results already obtained should be accompanied by an evaluation of the association between UPF consumption and health status, estimating the contribution within different dietary patterns.

Supplementary Materials: The following are available online at https:/ /www.mdpi.com/article/10 $.3390 /$ nu13082778/s1, Supplementary Table S1. Level of consumption of ultra-processed foods (UPF) expressed as percent energy provided by UPF intake in the total energy intake (TEI) in pregnant women and following different dietary patterns.

Author Contributions: Conceptualization, D.M. and C.D.B.; methodology, D.M.; formal analysis, M.M. and F.P.; investigation, M.M. and F.P.; writing—original draft preparation, M.M., D.M. and 
C.D.B.; writing-review and editing, P.R. and M.P.; visualization, M.M. and V.V.; supervision, D.M., C.D.B., P.R. and M.P. All authors have read and agreed to the published version of the manuscript.

Funding: This research received no external funding.

Data Availability Statement: The data presented in this study are available on request from the corresponding author.

Acknowledgments: D.M. and C.D.B. are grateful for the grant received from Piano di sostegno alla Ricerca-Linea 2, azione A-grant number PSR2020_DMART and PSR2020 CDELB. The authors thank the project MIND FoodS Hub (Milano Innovation District Food System Hub): Innovative concept for the eco-intensification of agricultural production and for the promotion of dietary patterns for human health and longevity through the creation in MIND of a digital Food System Hub (P.O.R. 2014-2020_BANDO Call HUB Ricerca e Innovazione_D.G.R. NR 727 del 5/11/2018).

Conflicts of Interest: The authors declare no conflict of interest.

\section{References}

1. Welch, R.W.; Mitchell, P.C. Food processing: A century of change. Br. Med. Bull. 2000, 56, 1-17. [CrossRef]

2. Kearney, J. Food consumption trends and drivers. Philos. Trans. R. Soc. B Biol. Sci. 2010, 365, 2793-2807. [CrossRef]

3. Slimani, N.; Deharveng, G.; Southgate, D.A.T.; Biessy, C.; Chajès, V.; van Bakel, M.M.E.; Boutron-Ruault, M.C.; McTaggart, A.; Grioni, S.; Verkaik-Kloosterman, J.; et al. Contribution of highly industrially processed foods to the nutrient intakes and patterns of middle-aged populations in the European Prospective Investigation into Cancer and Nutrition study. Eur. J. Clin. Nutr. 2009, 63, S206-S225. [CrossRef] [PubMed]

4. Monteiro, C.A.; Cannon, G.; Levy, R.; Moubarac, J.-C.; Jaime, P.; Martins, A.P.; Canella, D.; Louzada, M.; Parra, D. NOVA. The star shines bright. World Nutr. 2016, 7, 28-38.

5. Monteiro, C.A.; Cannon, G.; Levy, R.B.; Moubarac, J.C.; Louzada, M.L.; Rauber, F.; Khandpur, N.; Cediel, G.; Neri, D.; MartinezSteele, E.; et al. Ultra-processed foods: What they are and how to identify them. Public Health Nutr. 2019, 22, 936-941. [CrossRef]

6. Davidou, S.; Christodoulou, A.; Fardet, A.; Frank, K. The holistico-reductionist Siga classification according to the degree of food processing: An evaluation of ultra-processed foods in French supermarkets. Food Funct. 2020, 11, 2026-2039. [CrossRef] [PubMed]

7. Batal, M.; Johnson-Down, L.; Moubarac, J.C.; Ing, A.; Fediuk, K.; Sadik, T.; Tikhonov, C.; Chan, L.; Willows, N. Quantifying associations of the dietary share of ultra-processed foods with overall diet quality in First Nations peoples in the Canadian provinces of British Columbia, Alberta, Manitoba and Ontario. Public Health Nutr. 2018, 21, 103-113. [CrossRef] [PubMed]

8. Julia, C.; Martinez, L.; Allès, B.; Touvier, M.; Hercberg, S.; Méjean, C.; Kesse-Guyot, E. Contribution of ultra-processed foods in the diet of adults from the French NutriNet-Santé study. Public Health Nutr. 2018, 21, 27-37. [CrossRef]

9. Elizabeth, L.; Machado, P.; Zinöcker, M.; Baker, P.; Lawrence, M. Ultra-processed foods and health outcomes: A narrative review. Nutrients 2020, 12, 1955. [CrossRef]

10. Chen, X.; Zhang, Z.; Yang, H.; Qiu, P.; Wang, H.; Wang, F.; Zhao, Q.; Fang, J.; Nie, J. Consumption of ultra-processed foods and health outcomes: A systematic review of epidemiological studies. Nutr. J. 2020, 19. [CrossRef]

11. Pagliai, G.; Dinu, M.; Madarena, M.P.; Bonaccio, M.; Iacoviello, L.; Sofi, F. Consumption of ultra-processed foods and health status: A systematic review and meta-analysis. Br. J. Nutr. 2021, 125, 308-318. [CrossRef]

12. Hall, K.D.; Ayuketah, A.; Brychta, R.; Cai, H.; Cassimatis, T.; Chen, K.Y.; Chung, S.T.; Costa, E.; Courville, A.; Darcey, V.; et al. Ultra-Processed Diets Cause Excess Calorie Intake and Weight Gain: An Inpatient Randomized Controlled Trial of Ad Libitum Food Intake. Cell Metab. 2019, 30, 67-77.e3. [CrossRef]

13. Sadler, C.R.; Grassby, T.; Hart, K.; Raats, M.; Sokolović, M.; Timotijevic, L. Processed food classification: Conceptualisation and challenges. Trends Food Sci. Technol. 2021, 112, 149-162. [CrossRef]

14. Smiljanec, K.; Mbakwe, A.U.; Ramos-Gonzalez, M.; Mesbah, C.; Lennon, S.L. Associations of ultra-processed and unprocessed/minimally processed food consumption with peripheral and central hemodynamics, and arterial stiffness in young healthy adults. Nutrients 2020, 12, 3229. [CrossRef] [PubMed]

15. Beslay, M.; Srour, B.; Méjean, C.; Allès, B.; Fiolet, T.; Debras, C.; Chazelas, E.; Deschasaux, M.; Wendeu-Foyet, M.G.; Hercberg, S.; et al. Ultra-processed food intake in association with BMI change and risk of overweight and obesity: A prospective analysis of the French NutriNet-Santé cohort. PLoS Med. 2020, 17, e1003256. [CrossRef]

16. Costa de Miranda, R.; Rauber, F.; de Moraes, M.M.; Afonso, C.; Santos, C.; Rodrigues, S.; Levy, R.B. Consumption of ultraprocessed foods and non-communicable disease-related nutrient profile in Portuguese adults and elderly (2015-2016): The UPPER project. Br. J. Nutr. 2021, 125, 1177-1187. [CrossRef]

17. Oliveira, T.; Ribeiro, I.; Jurema-Santos, G.; Nobre, I.; Santos, R.; Rodrigues, C.; Oliveira, K.; Henrique, R.; Ferreira-e-Silva, W.; Araújo, A. Can the Consumption of Ultra-Processed Food Be Associated with Anthropometric Indicators of Obesity and Blood Pressure in Children 7 to 10 Years Old? Foods 2020, 9, 1567. [CrossRef] [PubMed]

18. Neri, D.; Martinez-Steele, E.; Monteiro, C.A.; Levy, R.B. Consumption of ultra-processed foods and its association with added sugar content in the diets of US children, NHANES 2009-2014. Pediatr. Obes. 2019, 14, e12563. [CrossRef] [PubMed] 
19. Onita, B.M.; Azeredo, C.M.; Jaime, P.C.; Levy, R.B.; Rauber, F. Eating context and its association with ultra-processed food consumption by British children. Appetite 2021, 157, 105007. [CrossRef]

20. Da Rocha, B.R.S.; Rico-Campà, A.; Romanos-Nanclares, A.; Ciriza, E.; Barbosa, K.B.F.; Martínez-González, M.Á.; Martín-Calvo, N. Adherence to Mediterranean diet is inversely associated with the consumption of ultra-processed foods among Spanish children: The SENDO project. Public Health Nutr. 2020. [CrossRef]

21. Vedovato, G.M.; Vilela, S.; Severo, M.; Rodrigues, S.; Lopes, C.; Oliveira, A. Ultra-processed food consumption, appetitive traits and BMI in children: A prospective study. Br. J. Nutr. 2021, 125, 1427-1436. [CrossRef]

22. Fangupo, L.J.; Haszard, J.J.; Taylor, B.J.; Gray, A.R.; Lawrence, J.A.; Taylor, R.W. Ultra-Processed Food Intake and Associations with Demographic Factors in Young New Zealand Children. J. Acad. Nutr. Diet. 2021, 121, 305-313. [CrossRef] [PubMed]

23. De Lacerda, A.T.; do Carmo, A.S.; de Sousa, T.M.; dos Santos, L.C. Participation of ultra-processed foods in Brazilian school children's diet and associated factors. Rev. Paul. Pediatr. 2020, 38, e2019034. [CrossRef]

24. Leffa, P.S.; Hoffman, D.J.; Rauber, F.; Sangalli, C.N.; Valmórbida, J.L.; Vitolo, M.R. Longitudinal associations between ultraprocessed foods and blood lipids in childhood. Br. J. Nutr. 2020, 124, 341-348. [CrossRef]

25. De Almeida Fonseca, P.C.; Ribeiro, S.A.V.; Andreoli, C.S.; de Carvalho, C.A.; Pessoa, M.C.; de Novaes, J.F.; Priore, S.E.; Franceschini, S.D.C.C. Association of exclusive breastfeeding duration with consumption of ultra-processed foods, fruit and vegetables in Brazilian children. Eur. J. Nutr. 2019, 58, 2887-2894. [CrossRef] [PubMed]

26. Ferreira, C.S.; Silva, D.A.; Gontijo, C.A.; Rinaldi, A.E.M. Consumption of minimally processed and ultra-processed foods among students from public and private schools. Rev. Paul. Pediatr. 2019, 37, 173-180. [CrossRef] [PubMed]

27. Batalha, M.A.; França, A.K.T.D.C.; Conceição, S.I.O.D.; Santos, A.M.D.; Silva, F.D.S.; Padilha, L.L.; Silva, A.A.M.D. Consumo de alimentos processados e ultraprocessados e fatores associados em crianças entre 13 e 35 meses de idade. Cad. Saude Publica 2017, 33, e00152016. [PubMed]

28. Bielemann, R.M.; Santos, L.P.; dos Santos Costa, C.; Matijasevich, A.; Santos, I.S. Early feeding practices and consumption of ultraprocessed foods at 6 y of age: Findings from the 2004 Pelotas (Brazil) Birth Cohort Study. Nutrition 2018, 47, 27-32. [CrossRef] [PubMed]

29. Karnopp, E.V.N.; Vaz, J.D.S.; Schafer, A.A.; Muniz, L.C.; Souza, R.D.L.V.D.; Santos, I.D.; Gigante, D.P.; Assunção, M.C.F. Consumo alimentar de crianças menores de seis anos conforme o grau de processamento. J. Pediatr. 2017, 93, 70-78. [CrossRef]

30. Sparrenberger, K.; Friedrich, R.R.; Schiffner, M.D.; Schuch, I.; Wagner, M.B. Ultra-processed food consumption in children from a Basic Health Unit. J. Pediatr. 2015, 91, 535-542. [CrossRef] [PubMed]

31. Melo, A.S.; Neves, F.S.; Batista, A.P.; Machado-Coelho, G.L.L.; Sartorelli, D.S.; de Faria, E.R.; Netto, M.P.; Oliveira, R.M.; Fontes, V.S.; Cândido, A.P.C. Percentage of energy contribution according to the degree of industrial food processing and associated factors in adolescents (EVA-JF study, Brazil). Public Health Nutr. 2021. [CrossRef] [PubMed]

32. Rocha, L.L.; Gratão, L.H.A.; do Carmo, A.S.; Costa, A.B.P.; de Freitas Cunha, C.; de Oliveira, T.R.P.R.; Mendes, L.L. School Type, Eating Habits, and Screen Time are Associated with Ultra-Processed Food Consumption among Brazilian Adolescents. J. Acad. Nutr. Diet. 2021, 121, 1136-1142. [CrossRef] [PubMed]

33. D'avila, H.F.; Kirsten, V.R. Energy intake from ultra-processed foods among adolescents. Rev. Paul. Pediatr. 2017, 35, 54-60. [CrossRef] [PubMed]

34. Oliveira, R.R.; Peter, N.B.; Muniz, L.C. Food consumption according to the level of processing among adolescents from the rural area of a municipality in the south of brazil. Cienc. Saude Coletiva 2021, 26, 1105-1114. [CrossRef]

35. Viola, P.C.D.A.F.; de Carvalho, C.A.; Bragança, M.L.B.M.; da Cunha França, A.K.T.; de Britto, M.T.S.S.; da Silva, A.A.M. High consumption of ultra-processed foods is associated with lower muscle mass in Brazilian adolescents in the RPS birth cohort. Nutrition 2020, 79-80, 110983. [CrossRef]

36. Rauber, F.; Martins, C.A.; Azeredo, C.M.; Leffa, P.S.; da Costa Louzada, M.L.; Levy, R.B. Eating context and ultraprocessed food consumption among UK adolescents. Br. J. Nutr. 2021, 1-11. [CrossRef]

37. Enes, C.C.; Camargo, C.M.D.; Justino, M.I.C. Ultra-processed food consumption and obesity in adolescents. Rev. Nutr. 2019, 32, e18170. [CrossRef]

38. Rohatgi, K.W.; Tinius, R.A.; Cade, W.T.; Steele, E.M.; Cahill, A.G.; Parra, D.C. Relationships between consumption of ultraprocessed foods, gestational weight gain and neonatal outcomes in a sample of US pregnant women. PeerJ 2017, 5, e4091. [CrossRef]

39. De Barros Gomes, C.; Malta, M.B.; da Costa Louzada, M.L.; Benício, M.H.D.A.; Barros, A.J.; Carvalhaes, M.A.D.B.L. Ultraprocessed Food Consumption by Pregnant Women: The Effect of an Educational Intervention with Health Professionals. Matern. Child Health J. 2019, 23, 692-703. [CrossRef]

40. Graciliano, N.G.; da Silveira, J.A.C.; de Oliveira, A.C.M. The consumption of ultra-processed foods reduces overall quality of diet in pregnant women. Cad. Saude Publica 2021, 37, e00030120. [CrossRef]

41. Paulino, D.S.M.; Pinho-Pompeu, M.; Assumpção, D.; Kasawara, K.T.; Surita, F.G. Dietary intake profile in high-risk pregnant women according to the degree of food processing. J. Matern. Neonatal Med. 2020. [CrossRef]

42. Silva, C.F.M.; Saunders, C.; Peres, W.; Folino, B.; Kamel, T.; dos Santos, M.S.; Padilha, P. Effect of ultra-processed foods consumption on glycemic control and gestational weight gain in pregnant with pregestational diabetes mellitus using carbohydrate counting. PeerJ 2021, 9, e10514. [CrossRef] [PubMed] 
43. Sandoval-Insausti, H.; Blanco-Rojo, R.; Graciani, A.; Lepez-Garcia, E.; Moreno-Franco, B.; Laclaustra, M.N.; Donat-Vargas, C.; OrdovÃis, J.M.; RodrÃ-Guez-Artalejo, F.; Guallar-Castillen, P. Ultra-processed Food Consumption and Incident Frailty: A Prospective Cohort Study of Older Adults. J. Gerontol. Ser. A Biol. Sci. Med. Sci. 2020, 75, 1126-1133. [CrossRef] [PubMed]

44. Sandoval-Insausti, H.; Jiménez-Onsurbe, M.; Donat-Vargas, C.; Rey-García, J.; Banegas, J.R.; Rodríguez-Artalejo, F.; GuallarCastillón, P. Ultra-processed food consumption is associated with abdominal obesity: A prospective cohort study in older adults. Nutrients 2020, 12, 2368. [CrossRef] [PubMed]

45. Pinho, M.G.M.; Lakerveld, J.; Harbers, M.C.; Sluijs, I.; Vermeulen, R.; Huss, A.; Boer, J.M.A.; Verschuren, W.M.M.; Brug, J.; Beulens, J.W.J.; et al. Ultra-processed food consumption patterns among older adults in the Netherlands and the role of the food environment. Eur. J. Nutr. 2020, 60, 2567-2580. [CrossRef]

46. Rauber, F.; da Costa Louzada, M.L.; Steele, E.M.; de Rezende, L.F.; Millett, C.; Monteiro, C.A.; Levy, R.B. Ultra-processed foods and excessive free sugar intake in the UK: A nationally representative cross-sectional study. BMJ Open 2019, 9, e027546. [CrossRef]

47. Rauber, F.; Chang, K.; Vamos, E.P.; da Costa Louzada, M.L.; Monteiro, C.A.; Millett, C.; Levy, R.B. Ultra-processed food consumption and risk of obesity: A prospective cohort study of UK Biobank. Eur. J. Nutr. 2021, 60, 2169-2180. [CrossRef]

48. Adams, J.; White, M. Characterisation of UK diets according to degree of food processing and associations with sociodemographics and obesity: Cross-sectional analysis of UK National Diet and Nutrition Survey (2008-2012). Int. J. Behav. Nutr. Phys. Act. 2015, 12, 160. [CrossRef]

49. Baraldi, L.G.; Martinez Steele, E.; Canella, D.S.; Monteiro, C.A. Consumption of ultra-processed foods and associated sociodemographic factors in the USA between 2007 and 2012: Evidence from a nationally representative cross-sectional study. BMJ Open 2018, 8, e020574. [CrossRef]

50. Martínez Steele, E.; Baraldi, L.G.; da Costa Louzada, M.L.; Moubarac, J.-C.; Mozaffarian, D.; Monteiro, C.A. Ultra-processed foods and added sugars in the US diet: Evidence from a nationally representative cross-sectional study. BMJ Open 2016, 6, e009892. [CrossRef]

51. Martínez Steele, E.; Khandpur, N.; da Costa Louzada, M.L.; Monteiro, C.A. Association between dietary contribution of ultraprocessed foods and urinary concentrations of phthalates and bisphenol in a nationally representative sample of the US population aged 6 years and older. PLoS ONE 2020, 15, e0236738. [CrossRef] [PubMed]

52. Juul, F.; Martinez-Steele, E.; Parekh, N.; Monteiro, C.A.; Chang, V.W. Ultra-processed food consumption and excess weight among US adults. Br. J. Nutr. 2018, 120, 90-100. [CrossRef] [PubMed]

53. Rauber, F.; da Costa Louzada, M.L.; Steele, E.; Millett, C.; Monteiro, C.A.; Levy, R.B. Ultra-Processed Food Consumption and Chronic Non-Communicable Diseases-Related Dietary Nutrient Profile in the UK (2008-2014). Nutrients 2018, 10, 587. [CrossRef] [PubMed]

54. Rauber, F.; Steele, E.M.; da Costa Louzada, M.L.; Millett, C.; Monteiro, C.A.; Levy, R.B. Ultra-processed food consumption and indicators of obesity in the United Kingdom population (2008-2016). PLoS ONE 2020, 15, e0232676. [CrossRef]

55. Bonaccio, M.; di Castelnuovo, A.; Costanzo, S.; De Curtis, A.; Persichillo, M.; Sofi, F.; Cerletti, C.; Donati, M.B.; De Gaetano, G.; Iacoviello, L. Ultra-processed food consumption is associated with increased risk of all-cause and cardiovascular mortality in the Moli-sani Study on behalf of the Moli-sani Study Investigators. Am. J. Clin. Nutr 2021, 113, 446-455. [CrossRef]

56. Dinu, M.; Bonaccio, M.; Martini, D.; Madarena, M.P.; Vitale, M.; Pagliai, G.; Esposito, S.; Ferraris, C.; Guglielmetti, M.; Rosi, A.; et al. Reproducibility and validity of a food-frequency questionnaire (NFFQ) to assess food consumption based on the NOVA classification in adults. Int. J. Food Sci. Nutr. 2021. [CrossRef]

57. Da Conceição, A.R.; De Almeida Fonseca, P.C.; De Castro Morais, D.; De Souza, E.C.G. Association of the degree of food processing with the consumption of nutrients and blood pressure. Mundo Saude 2019, 43, 512-529. [CrossRef]

58. Bielemann, R.M.; Santos Motta, J.V.; Minten, G.C.; Horta, B.L.; Gigante, D.P. Consumption of ultra-processed foods and their impact on the diet of young adults. Rev. Saude Publica 2015, 49, 28. [CrossRef]

59. Zhong, G.-C.; Gu, H.-T.; Peng, Y.; Wang, K.; Wu, Y.-Q.-L.; Hu, T.-Y.; Jing, F.-C.; Hao, F.-B. Association of ultra-processed food consumption with cardiovascular mortality in the US population: Long-term results from a large prospective multicenter study. Int. J. Behav. Nutr. Phys. Act. 2021, 18, 21. [CrossRef]

60. Costa, C.D.S.; Assunção, M.C.F.; Loret de Mola, C.; Cardoso, J.D.S.; Matijasevich, A.; Barros, A.J.D.; Santos, I.S. Role of ultraprocessed food in fat mass index between 6 and 11 years of age: A cohort study. Int. J. Epidemiol. 2021, 50, 256-265. [CrossRef]

61. Da Silva, D.C.G.; Ferreira, F.G.; Pereira, D.L.M.; de Magalhães, E.L.G.; Longo, G.Z. Degree of food processing and its relationship with overweight and body adiposity in Brazilian adults. Rev. Nutr. 2021, 34, 1-11. [CrossRef] [PubMed]

62. Da Silva Scaranni, P.D.O.; de Oliveira Cardoso, L.; Chor, D.; Melo, E.C.P.; Matos, S.M.A.; Giatti, L.; Barreto, S.M.; da Fonseca, M.D.J.M. Ultra-processed foods, changes in blood pressure and incidence of hypertension: The Brazilian Longitudinal Study of Adult Health (ELSA-Brasil). Public Health Nutr. 2021, 24, 3352-3360. [CrossRef] [PubMed]

63. Cattafesta, M.; Petarli, G.B.; Zandonade, E.; Bezerra, O.M.D.P.A.; Abreu, S.M.R.D.; Salaroli, L.B. Energy contribution of NOVA food groups and the nutritional profile of the Brazilian rural workers' diets. PLoS ONE 2020, 15, e0240756. [CrossRef] [PubMed]

64. Souza, A.M.; Bezerra, I.W.L.; Pereira, G.S.; Torres, K.G.; Costa, R.M.; Oliveira, A.G. Relationships between Motivations for Food Choices and Consumption of Food Groups: A Prospective Cross-Sectional Survey in Manufacturing Workers in Brazil. Nutrients 2020, 12, 1490. [CrossRef] 
65. Smaira, F.I.; Mazzolani, B.C.; Peçanha, T.; dos Santos, K.M.; Rezende, D.A.N.; Araujo, M.E.; Bonfiglioli, K.; Scagliusi, F.B.; Benatti, F.B.; de Sá Pinto, A.L.; et al. Ultra-processed food consumption associates with higher cardiovascular risk in rheumatoid arthritis. Clin. Rheumatol. 2020, 39, 1423-1428. [CrossRef] [PubMed]

66. Canhada, S.L.; Luft, V.C.; Giatti, L.; Duncan, B.B.; Chor, D.; Maria de Jesus, M.; Matos, S.M.A.; Molina, M.D.C.B.; Barreto, S.M.; Levy, R.B.; et al. Ultra-processed foods, incident overweight and obesity, and longitudinal changes in weight and waist circumference: The Brazilian Longitudinal Study of Adult Health (ELSA-Brasil). Public Health Nutr. 2020, 23, 1076-1086. [CrossRef]

67. Silverio, R.N.C.; de Aquino Lacerda, E.M.; Fortins, R.F.; de Lima, G.C.F.; Scancetti, L.B.; do Carmo, C.N.; da Cunha, L.V.S.; Luescher, J.L.; de Carvalho Padilha, P. Predictive factors of non-HDL cholesterol in children and adolescents with type 1 diabetes mellitius: A cross-sectional study. Diabetes Res. Clin. Pract. 2019, 154, 9-16. [CrossRef]

68. Sousa, R.D.S.; Bragança, M.L.B.M.; Oliveira, B.R.D.; Coelho, C.C.N.D.S.; Silva, A.A.M.D. Association between the Degree of Processing of Consumed Foods and Sleep Quality in Adolescents. Nutrients 2020, 12, 462. [CrossRef]

69. Longo, A.; Ribas, B.; Weber, B.; Bertoldi, E.; Borges, L.; Abib, R. Consumption of foods according to their degree of processing in patients with established atherosclerosis disease. Rev. Chil. Nutr. 2020, 47, 351-358. [CrossRef]

70. Rezende-Alves, K.; Hermsdorff, H.H.M.; Miranda, A.E.D.S.; Lopes, A.C.S.; Bressan, J.; Pimenta, A.M. Food processing and risk of hypertension: Cohort of Universities of Minas Gerais, Brazil (CUME Project). Public Health Nutr. 2020. [CrossRef]

71. Ferreira Fortins, R.; de Aquino Lacerda, E.M.; Silverio, R.N.C.; do Carmo, C.N.; Ferreira, A.A.; Felizardo, C.; do Nascimento, B.F.; Luescher, J.L.; de Carvalho Padilha, P. Predictor factors of glycemic control in children and adolescents with type 1 diabetes mellitus treated at a referral service in Rio de Janeiro, Brazil. Diabetes Res. Clin. Pract. 2019, 154, 138-145. [CrossRef]

72. Silva, F.M.; Giatti, L.; de Figueiredo, R.C.; Molina, M.D.C.B.; de Oliveira Cardoso, L.; Duncan, B.B.; Barreto, S.M. Consumption of ultra-processed food and obesity: Cross sectional results from the Brazilian Longitudinal Study of Adult Health (ELSA-Brasil) cohort (2008-2010). Public Health Nutr. 2018, 21, 2271-2279. [CrossRef]

73. Dos Santos Simões, B.; Barreto, S.M.; Del Carmen Bisi Molina, M.; Luft, V.C.; Duncan, B.B.; Schmidt, M.I.; Benseñor, I.J.M.; de Oliveira Cardoso, L.; Levy, R.B.; Giatti, L. Consumption of ultra-processed foods and socioeconomic position: A cross-sectional analysis of the Brazilian Longitudinal Study of Adult Health. Cad. Saude Publica 2018, 34, e00019717.

74. Da Costa Louzada, M.L.; Ricardo, C.Z.; Steele, E.M.; Levy, R.B.; Cannon, G.; Monteiro, C.A. The share of ultra-processed foods determines the overall nutritional quality of diets in Brazil. Public Health Nutr. 2018, 21, 94-102. [CrossRef]

75. Da Costa Louzada, M.L.; Martins, A.P.B.; Canella, D.S.; Baraldi, L.G.; Levy, R.B.; Claro, R.M.; Moubarac, J.-C.; Cannon, G.; Monteiro, C.A. Impact of ultra-processed foods on micronutrient content in the Brazilian diet. Rev. Saude Publica 2015, 49, 1-8. [CrossRef] [PubMed]

76. Da Costa Louzada, M.L.; Baraldi, L.G.; Steele, E.M.; Martins, A.P.B.; Canella, D.S.; Moubarac, J.-C.; Levy, R.B.; Cannon, G.; Afshin, A.; Imamura, F.; et al. Consumption of ultra-processed foods and obesity in Brazilian adolescents and adults. Prev. Med. 2015, 81, 9-15. [CrossRef]

77. Baraldi, L.G.; Steele, E.M.; Da Costa Louzada, M.L.; Monteiro, C.A. Associations between ultraprocessed food consumption and total water intake in the US population. J. Acad. Nutr. Diet. 2021, 121, 1695-1703. [CrossRef] [PubMed]

78. Bidinotto, A.B.; Martinez-Steele, E.; Cunha-Cruz, J.; Thomson, W.M.; Hugo, F.N.; Hilgert, J.B. Food processing and its association with dental caries: Data from NHANES 2011-2014. Community Dent. Oral Epidemiol. 2021. [CrossRef]

79. Yang, Q.; Zhang, Z.; Steele, E.M.; Moore, L.V.; Jackson, S.L. Ultra-Processed Foods and Excess Heart Age among U.S. Adults. Am. J. Prev. Med. 2020, 59, e197-e206. [CrossRef]

80. Zheng, L.; Sun, J.; Yu, X.; Zhang, D. Ultra-Processed Food Is Positively Associated with Depressive Symptoms among United States Adults. Front. Nutr. 2020, 7, 600449. [CrossRef]

81. Gupta, S.; Rose, C.M.; Buszkiewicz, J.; Ko, L.K.; Mou, J.; Cook, A.; Aggarwal, A.; Drewnowski, A. Characterising percentage energy from ultra-processed foods by participant demographics, diet quality and diet cost: Findings from the Seattle Obesity Study (SOS) III. Br. J. Nutr. 2020, 773-781. [CrossRef]

82. Zhang, Z.; Jackson, S.L.; Martinez, E.; Gillespie, C.; Yang, Q. Association between ultraprocessed food intake and cardiovascular health in US adults: A cross-sectional analysis of the NHANES 2011-2016. Am. J. Clin. Nutr. 2021, 113, 428-436. [CrossRef]

83. Martínez Steele, E.; Juul, F.; Neri, D.; Rauber, F.; Monteiro, C.A. Dietary share of ultra-processed foods and metabolic syndrome in the US adult population. Prev. Med. 2019, 125, 40-48. [CrossRef]

84. Andrade, G.C.; Julia, C.; Deschamps, V.; Srour, B.; Hercberg, S.; Kesse-Guyot, E.; Allès, B.; Chazelas, E.; Deschasaux, M.; Touvier, M.; et al. Consumption of ultra-processed food and its association with sociodemographic characteristics and diet quality in a representative sample of French adults. Nutrients 2021, 13, 682. [CrossRef] [PubMed]

85. Gehring, J.; Touvier, M.; Baudry, J.; Julia, C.; Buscail, C.; Srour, B.; Hercberg, S.; Péneau, S.; Kesse-Guyot, E.; Allès, B. The consumption of ultra-processed foods by fish-eaters, vegetarians and vegans is associated with the duration and commencing age of diet. Proc. Nutr. Soc. 2020, 79, 15-18. [CrossRef]

86. Gehring, J.; Touvier, M.; Baudry, J.; Julia, C.; Buscail, C.; Srour, B.; Hercberg, S.; Péneau, S.; Kesse-Guyot, E.; Allès, B. Consumption of Ultra-Processed Foods by Pesco-Vegetarians, Vegetarians, and Vegans: Associations with Duration and Age at Diet Initiation. J. Nutr. 2021, 151, 120-131. [CrossRef] 
87. Srour, B.; Fezeu, L.K.; Kesse-Guyot, E.; Allès, B.; Debras, C.; Druesne-Pecollo, N.; Chazelas, E.; Deschasaux, M.; Hercberg, S.; Galan, P.; et al. Ultraprocessed Food Consumption and Risk of Type 2 Diabetes among Participants of the NutriNet-Santé Prospective Cohort. JAMA Intern. Med. 2020, 180, 283. [CrossRef]

88. Vasseur, P.; Dugelay, E.; Benamouzig, R.; Savoye, G.; Lan, A.; Srour, B.; Hercberg, S.; Touvier, M.; Hugot, J.P.; Julia, C.; et al. Dietary Patterns, Ultra-processed Food, and the Risk of Inflammatory Bowel Diseases in the NutriNet-Santé Cohort. Inflamm. Bowel Dis. 2021, 27, 65-73. [CrossRef]

89. Schnabel, L.; Kesse-Guyot, E.; Allès, B.; Touvier, M.; Srour, B.; Hercberg, S.; Buscail, C.; Julia, C. Association Between Ultraprocessed Food Consumption and Risk of Mortality among Middle-aged Adults in France. JAMA Intern. Med. 2019, $179,490$. [CrossRef]

90. Adjibade, M.; Julia, C.; Allès, B.; Touvier, M.; Lemogne, C.; Srour, B.; Hercberg, S.; Galan, P.; Assmann, K.E.; Kesse-Guyot, E. Prospective association between ultra-processed food consumption and incident depressive symptoms in the French NutriNetSanté cohort. BMC Med. 2019, 17, 78. [CrossRef] [PubMed]

91. Schnabel, L.; Buscail, C.; Sabate, J.M.; Bouchoucha, M.; Kesse-Guyot, E.; Allès, B.; Touvier, M.; Monteiro, C.A.; Hercberg, S.; Benamouzig, R.; et al. Association between Ultra-Processed Food Consumption and Functional Gastrointestinal Disorders: Results from the French NutriNet-Santé Cohort. Am. J. Gastroenterol. 2018, 113, 1217-1228. [CrossRef]

92. Polsky, J.Y.; Moubarac, J.C.; Garriguet, D. Consumption of ultra-processed foods in Canada. Health Rep. 2020, 31, 3-15. [PubMed]

93. Nardocci, M.; Polsky, J.Y.; Moubarac, J.C. Consumption of ultra-processed foods is associated with obesity, diabetes and hypertension in Canadian adults. Can. J. Public Health 2021, 112, 421-429. [CrossRef]

94. Batal, M.; Johnson-Down, L.; Moubarac, J.-C.C.; Ing, A.; Fediuk, K.; Sadik, T.; Chan, H.M.; Willows, N. Sociodemographic associations of the dietary proportion of ultra-processed foods in First Nations peoples in the Canadian provinces of British Columbia, Manitoba, Alberta and Ontario. Int. J. Food Sci. Nutr. 2018, 69, 753-761. [CrossRef]

95. Nardocci, M.; Leclerc, B.S.; Louzada, M.L.; Monteiro, C.A.; Batal, M.; Moubarac, J.C. Consumption of ultra-processed foods and obesity in Canada. Can. J. Public Health 2019, 110, 4-14. [CrossRef]

96. Moubarac, J.C.; Batal, M.; Louzada, M.L.; Steele, E.M.; Monteiro, C.A. Consumption of ultra-processed foods predicts diet quality in Canada. Appetite 2017, 108, 512-520. [CrossRef]

97. Blanco-Rojo, R.; Sandoval-Insausti, H.; López-Garcia, E.; Graciani, A.; Ordovás, J.M.; Banegas, J.R.; Rodríguez-Artalejo, F.; Guallar-Castillón, P. Consumption of Ultra-Processed Foods and Mortality: A National Prospective Cohort in Spain. Mayo Clin. Proc. 2019, 94, 2178-2188. [CrossRef] [PubMed]

98. Asma', A.; Gan, H.J.; Hayati, M.Y.; Khairil-Shazmin, K.; Zainudin, A.A. Food classification system based on food processing and its relationship with nutritional status of adults in Terengganu, Malaysia. Food Res. 2019, 4, 539-546.

99. Khasbullah, N.A.; Ahmad, F.T.; Yusof, H.M. Ultra-processed Food Consumption in Relation to BMI and Body Fat Percentage of Adults in Terengganu. Malays. J. Med. Health Sci. 2020, 16, 37-43.

100. Asma', A.; Wan Syakirah Alia, W.M.; Aziz, Y.; Hayati, M.Y. Energy contribution of NOVA food groups and socio-demographic determinants of ultra-processed groups among adults in Terengganu, Malaysia. Food Res. 2019, 3, $640-648$.

101. Machado, P.P.; Steele, E.M.; Levy, R.B.; da Costa Louzada, M.L.; Rangan, A.; Woods, J.; Gill, T.; Scrinis, G.; Monteiro, C.A. Ultra-processed food consumption and obesity in the Australian adult population. Nutr. Diabetes 2020, 10, 39. [CrossRef]

102. Machado, P.P.; Steele, E.M.; Levy, R.B.; Sui, Z.; Rangan, A.; Woods, J.; Gill, T.; Scrinis, G.; Monteiro, C.A. Ultra-processed foods and recommended intake levels of nutrients linked to non-communicable diseases in Australia: Evidence from a nationally representative cross-sectional study. BMJ Open 2019, 9, e029544. [CrossRef]

103. Machado, P.P.; Steele, E.M.; da Costa Louzada, M.L.; Levy, R.B.; Rangan, A.; Woods, J.; Gill, T.; Scrinis, G.; Monteiro, C.A. Ultra-processed food consumption drives excessive free sugar intake among all age groups in Australia. Eur. J. Nutr. 2020, 59, 2783-2792. [CrossRef]

104. Vandevijvere, S.; Pedroni, C.; De Ridder, K.; Castetbon, K. The cost of diets according to their caloric share of ultraprocessed and minimally processed foods in belgium. Nutrients 2020, 12, 2787. [CrossRef]

105. Khandpur, N.; Cediel, G.; Obando, D.A.; Jaime, P.C.; Parra, D.C. Sociodemographic factors associated with the consumption of ultra-processed foods in Colombia. Rev. Saude Publica 2020, 54, 19. [CrossRef]

106. Parra, D.C.; Da Costa Louzada, M.L.; Moubarac, J.C.; Bertazzi-Levy, R.; Khandpur, N.; Cediel, G.; Monteiro, C.A. Association between ultra-processed food consumption and the nutrient profile of the Colombian diet in 2005. Salud Publica Mex. 2019, 61, 147-154. [CrossRef]

107. Monge, A.; Silva Canella, D.; López-Olmedo, N.; Lajous, M.; Cortés-Valencia, A.; Stern, D. Ultraprocessed beverages and processed meats increase the incidence of hypertension in Mexican women. Br. J. Nutr. 2020, 126, 600-611. [CrossRef] [PubMed]

108. Marrón-Ponce, J.A.; Flores, M.; Cediel, G.; Monteiro, C.A.; Batis, C. Associations between Consumption of Ultra-Processed Foods and Intake of Nutrients Related to Chronic Non-Communicable Diseases in Mexico. J. Acad. Nutr. Diet. 2019, 119, $1852-1865$. [CrossRef] [PubMed]

109. Marrón-Ponce, J.A.; Sánchez-Pimienta, T.G.; Da Costa Louzada, M.L.; Batis, C. Energy contribution of NOVA food groups and sociodemographic determinants of ultra-processed food consumption in the Mexican population. Public Health Nutr. 2018, 21, 87-93. [CrossRef] [PubMed]

110. Shim, J.-S.; Shim, S.-Y.; Cha, H.-J.; Kim, J.; Kim, H.C. Socioeconomic Characteristics and Trends in the Consumption of UltraProcessed Foods in Korea from 2010 to 2018. Nutrients 2021, 13, 1120. [CrossRef] 
111. Sung, H.; Park, J.M.; Oh, S.U.; Ha, K.; Joung, H. Consumption of Ultra-Processed Foods Increases the Likelihood of Having Obesity in Korean Women. Nutrients 2021, 13, 698. [CrossRef]

112. Cediel, G.; Reyes, M.; Da Costa Louzada, M.L.; Martinez Steele, E.; Monteiro, C.A.; Corvalán, C.; Uauy, R. Ultra-processed foods and added sugars in the Chilean diet (2010). Public Health Nutr. 2018, 21, 125-133. [CrossRef] [PubMed]

113. Cediel, G.; Reyes, M.; Corvalán, C.; Levy, R.B.; Uauy, R.; Monteiro, C.A. Ultra-processed foods drive to unhealthy diets: Evidence from Chile. Public Health Nutr. 2020, 24, 1698-1707. [CrossRef] [PubMed]

114. Fliss-Isakov, N.; Zelber-Sagi, S.; Ivancovsky-Wajcman, D.; Shibolet, O.; Kariv, R. Ultra-processed food intake and smoking interact in relation with colorectal adenomas. Nutrients 2020, 12, 3507. [CrossRef]

115. Koiwai, K.; Takemi, Y.; Hayashi, F.; Ogata, H.; Matsumoto, S.; Ozawa, K.; Machado, P.P.; Monteiro, C.A. Consumption of ultra-processed foods decreases the quality of the overall diet of middle-aged Japanese adults. Public Health Nutr. 2019, 22, 2999-3008. [CrossRef]

116. Setyowati, D.; Andarwulan, N.; Giriwono, P.E. Processed and ultraprocessed food consumption pattern in the Jakarta Individual Food Consumption Survey 2014. Asia Pac. J. Clin. Nutr. 2018, 27, 840-847.

117. Nasreddine, L.; Tamim, H.; Itani, L.; Nasrallah, M.P.; Isma'Eel, H.; Nakhoul, N.F.; Abou-Rizk, J.; Naja, F. A minimally processed dietary pattern is associated with lower odds of metabolic syndrome among Lebanese adults. Public Health Nutr. 2018, 21, 160-171. [CrossRef] [PubMed]

118. Khandpur, N.; Neri, D.A.; Monteiro, C.; Mazur, A.; Frelut, M.-L.; Boyland, E.; Weghuber, D.; Thivel, D. Ultra-Processed Food Consumption among the Paediatric Population: An Overview and Call to Action from the European Childhood Obesity Group. Ann. Nutr. Metab. 2020, 76, 109-113. [CrossRef]

119. Vandevijvere, S.; De Ridder, K.; Fiolet, T.; Bel, S.; Tafforeau, J. Consumption of ultra-processed food products and diet quality among children, adolescents and adults in Belgium. Eur. J. Nutr. 2019, 58, 3267-3278. [CrossRef] [PubMed]

120. Da Silva Cruz Lopes, A.E.; Araújo, L.F.; Levy, R.B.; Barreto, S.M.; Giatti, L. Association between consumption of ultra-processed foods and serum C-reactive protein levels: Cross-sectional results from the ELSA-Brasil study. Sao Paulo Med. J. 2019, 137, 169-176. [CrossRef] [PubMed]

121. Fiolet, T.; Srour, B.; Sellem, L.; Kesse-Guyot, E.; Allès, B.; Méjean, C.; Deschasaux, M.; Fassier, P.; Latino-Martel, P.; Beslay, M.; et al. Consumption of ultra-processed foods and cancer risk: Results from NutriNet-Santé prospective cohort. BMJ 2018, $360, \mathrm{k} 322$. [CrossRef] [PubMed]

122. Griffin, J.; Albaloul, A.; Kopytek, A.; Elliott, P.; Frost, G. Effect of ultraprocessed food intake on cardiometabolic risk is mediated by diet quality: A cross-sectional study. BMJ Nutr. Prev. Health 2021. [CrossRef]

123. Da Silva, A.; Brum Felício, M.; Caldas, A.P.S.; Hermsdorff, H.H.; Torreglosa, C.R.; Bersch-Ferreira, Â.C.; Weber, B.; Marcadenti, A.; Bressan, J. Ultra-processed foods consumption is associated with cardiovascular disease and cardiometabolic risk factors in Brazilians with established cardiovascular events. Int. J. Food Sci. Nutr. 2021. [CrossRef] [PubMed]

124. Vandevijvere, S.; Jaacks, L.M.; Monteiro, C.A.; Moubarac, J.C.; Girling-Butcher, M.; Lee, A.C.; Pan, A.; Bentham, J.; Swinburn, B Global trends in ultraprocessed food and drink product sales and their association with adult body mass index trajectories. Obes. Rev. 2019, 20, 10-19. [CrossRef]

125. Martines, R.M.; Machado, P.P.; Neri, D.A.; Levy, R.B.; Rauber, F. Association between watching TV whilst eating and children's consumption of ultraprocessed foods in United Kingdom. Matern. Child. Nutr. 2019, 15, e12819. [CrossRef] [PubMed]

126. Smith, A.D.A.C.; Emmett, P.M.; Newby, P.K.; Northstone, K. A comparison of dietary patterns derived by cluster and principal components analysis in a UK cohort of children. Eur. J. Clin. Nutr. 2011, 65, 1102-1109. [CrossRef] [PubMed]

127. Andrade, G.C.; Gombi-Vaca, M.F.; da Costa Louzada, M.L.; Azeredo, C.M.; Levy, R.B. The consumption of ultra-processed foods according to eating out occasions. Public Health Nutr. 2020, 23, 1041-1048. [CrossRef]

128. Donat-Vargas, C.; Sandoval-Insausti, H.; Rey-García, J.; Moreno-Franco, B.; Åkesson, A.; Banegas, J.R.; Rodríguez-Artalejo, F.; Guallar-Castillón, P. High Consumption of Ultra-Processed Food is Associated with Incident Dyslipidemia: A Prospective Study of Older Adults. J. Nutr. 2021. [CrossRef]

129. Orlich, M.J.; Jaceldo-Siegl, K.; Sabaté, J.; Fan, J.; Singh, P.N.; Fraser, G.E. Patterns of food consumption among vegetarians and non-vegetarians. Br. J. Nutr. 2014, 112, 1644-1653. [CrossRef] [PubMed]

130. Food and Agriculture Organization of the United Nations (FAO). Dietary Assessment: A Resource Guide to Method Selection and Application in Low Resource Settings; FAO: Rome, Italy, 2018; ISBN 978-92-5-130635-2.

131. Riboli, E. Nutrition and cancer: Background and rationale of the European Prospective Investigation into Cancer and Nutrition (EPIC). Ann. Oncol. 1992, 3, 783-791. [CrossRef] [PubMed]

132. Gibney, M.J.; Forde, C.G.; Mullally, D.; Gibney, E.R. Ultra-processed foods in human health: A critical appraisal. Am. J. Clin. Nutr. 2017, 106, 717-724. [CrossRef] [PubMed]

133. Jones, J.M. Food processing: Criteria for dietary guidance and public health? Proc. Nutr. Soc. 2019, 78, 4-18. [CrossRef] [PubMed] 